\title{
Determination of a System's Entropy Using Pyroelectric Sensors
}

\author{
Angel Cuadras, Member, IEEE, and Victoria Julia Ovejas
}

\begin{abstract}
We propose a system for measuring entropy variations, $\Delta S$, in thermal systems using pyroelectric sensors. These sensors convert time-dependent temperature variations into electrical current. Consequently, heat and temperature variations are obtained, and sensor entropy is inferred. Various polyvinylidene fluoride and lead zirconate titanate sensors have been tested. Two types of measurements are performed. One in the volume, to measure entropy variations in a heat source, and the other on the surface, to measure entropy flux delivered by the heat source. Thermodynamic models and heat transfer dynamic simulations agree with the experimental results and relate the sensor entropy to the heat source entropy. These results show that pyroelectric sensors can enable entropy monitoring of thermal processes to improve system performance.
\end{abstract}

Index Terms-Entropy, heat, pyroelectric sensor, temperature.

\section{INTRODUCTION}

I $\mathrm{N}$ ENERGY generation systems, output energy is the target to be maximized, whereas energy efficiency must retain the maximum yield. However, losses during energy conversion can reach up to $60 \%$, with most of it being dissipated as low-grade thermal energy [1]. For instance, in electrical energy generation, gas, wind, and water turbine efficiencies are as large as $40 \%, 60 \%$, and $90 \%$, respectively. The efficiency is around $30 \%-60 \%$ in electrical motors, $10 \%-50 \%$ in combustion engines, and $20 \%-50 \%$ in refrigerators. Consequently, energy conversion efficiency is a critical issue that must be addressed, i.e., the output energy for a given input energy should be maximized.

Energy loss is connected to entropy generation. Entropy is a common parameter in chemical reactions and is commonly studied in thermodynamics [2], [3], biology [4], and materials science [5]-[7]. From a technical viewpoint, entropy is considered in the design of refrigeration and combustion engines for thermodynamic efficiency [2], [8] as well as in batteries [9], [10]. However, entropy generation in irreversible

Manuscript received June 16, 2015; revised July 30, 2015; accepted August 3, 2015. This work was supported by the Ministerio de Ciencia e Innovación through the Spanish Government under Contract TEC2011-27397. The work of V. J. Ovejas was supported by the Ministerio de Educación, Cultura y Deporte through the Formación del Profesorado Universitario. This is an expanded paper from the IEEE SENSORS 200X Conference. The associate editor coordinating the review of this paper and approving it for publication was Prof. Zeynep Celik-Butler.

The authors are with the Department of Electronic Engineering, Escola d'Enginyeria de Telecomunicació i Aeronàutica de Castelldefels, Universitat Politècnica de Catalunya, Barcelona 08034, Spain (e-mail: angel.cuadras@upc.edu; victoria.julia.ovejas@upc.edu).

Color versions of one or more of the figures in this paper are available online at http://ieeexplore.ieee.org.

Digital Object Identifier 10.1109/JSEN.2015.2466112 processes is not commonly monitored in real time, although it can be interesting to do so in many fields ranging from thermal energy management to aging systems [2], [11], [12]. In our previous research [13], [14], we found that entropy can be used to estimate the failure and aging of an electrical circuit because it increases monotonously; thus, a threshold for maximum tolerated wear out, either for electrical or mechanical systems, can be defined.

Heat and temperature measurements are needed to estimate entropy. Heat is usually measured using calorimeters, which are not practical for in situ measurements. Heat flux sensors based on the Seebeck effect are also being used. With respect to temperature, common sensors are thermistors, thermocouples, RTDs, and pyroelectric sensors. Thermistors and RTDs are semiconductors and conductors, respectively, whose resistances are temperature dependent. Thermocouples are based on the Seebeck effect, and pyroelectric sensors are sensitive to time-dependent temperature fluctuations. Our approach is to relate temperature and heat in the sensor through the thermal capacity, $C_{\mathrm{p}}=\mathrm{dQ} / \mathrm{dT}$. Among the possible sensors, pyroelectric sensors are preferred because they are bulk sensors with a well-defined heat capacity and need no external bias. Thermocouples were discarded because they are junctions of two materials, and RTDs and thermistors need to be biased. Pyroelectric sensors convert temperature variations into electrical currents; they have been widely commercialized as presence detectors, fire detectors, and infrared detectors [16]-[19].

In this work, we investigate the use of pyroelectric sensors in the monitoring of temperature fluctuations, obtain the heat involved in the process via heat capacity, and estimate the sensor entropy variation. We consider entropy measurements in volumes and entropy flux through surfaces (a preliminary study was presented in [15]). Sensor entropy is finally related to entropy change in the system under study using a convenient model. This paper is organized as follows. In Section II, a phenomenological model describing the relationship between the basic heat conduction equation, thermodynamics, and pyroelectric current generation is described. In Section III, we describe the materials and methods used to test our hypothesis. In Section IV, we report on the entropy measured using various pyroelectric sensors; finally, we discuss our results and summarize the conclusions.

\section{THEORETICAL APPROACH}

The aim of this research is to measure entropy variation in a heat source using pyroelectric sensors. These sensors 


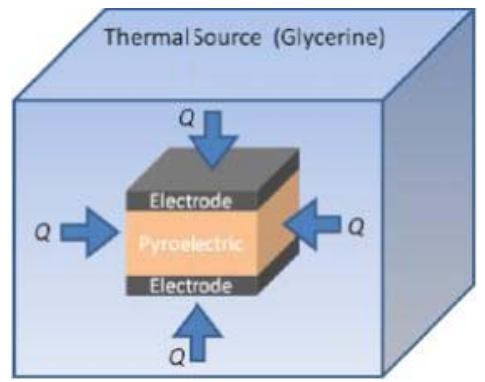

Fig. 1. Pyroelectric sensor within a heat source for measuring internal $\Delta \mathrm{S}$.

generate electrical currents proportional to temperature fluctuations. Therefore, we investigate how thermal energy is transferred from the heat source to the pyroelectric sensor. This is evaluated using heat transfer equations.

\section{A. Entropy and Heat Transfer}

In thermodynamics [2], [3], entropy, $S$, is a function of state: intrinsic and extensive. The change in $S$ is defined as the heat exchanged by the material, $\delta Q$, at a particular temperature, $T$.

$$
d S=\frac{\delta Q}{T} .
$$

To study entropy variations using a pyroelectric sensor, we consider both heat exchange and temperature variations. Thus, an entropy sensor must monitor both temperature and heat.

Heat is transferred from the environment into a pyroelectric sensor by conduction at a temperature, $T$, as illustrated in Fig. 1. This process is ruled by the general heat conduction equation for solids.

$$
\rho C_{p} \frac{\partial T}{\partial t}=\nabla \cdot(k \nabla T)+\mathrm{Q},
$$

where $\rho, C_{\mathrm{P}}$, and $k$ are the density, heat capacity at constant pressure $\left(C_{\mathrm{p}}=c_{\mathrm{p}} \cdot m\right.$, where $m$ is the mass and $c_{\mathrm{p}}$ is the specific heat capacity), and thermal conductivity of the pyroelectric material, respectively [20]; $t$ is the time, and $Q$ represents environmental heat sources. The term on the left-hand side of the equation describes time-dependent thermal variation, whereas that on the right-hand side is related to the thermal spatial gradients. From this definition, we can see that pyroelectric sensors can be used to characterize heat transfer (this observation will be clarified when studying pyroelectric response: see Eq. (3) [2], [21]).

There are certain considerations regarding the boundary conditions of this equation that should be noted:

i) Heat transfer mechanisms. In solids, heat is transferred by conduction. Convection is considered as a boundary condition at the interface if the sensor is in contact with fluids. Radiation is negligible for solid/liquid interfaces at low temperatures $(\mathrm{T}<340 \mathrm{~K})$.

ii) Space vs. time heat transfer. The contributions of both terms need to be considered. To discriminate between each term's contribution, simulations are performed.

iii) Volume vs. surface measurements. The entropy sensor can be located either inside a medium for volume entropy measurement (as illustrated in Fig. 1) or fixed to a surface

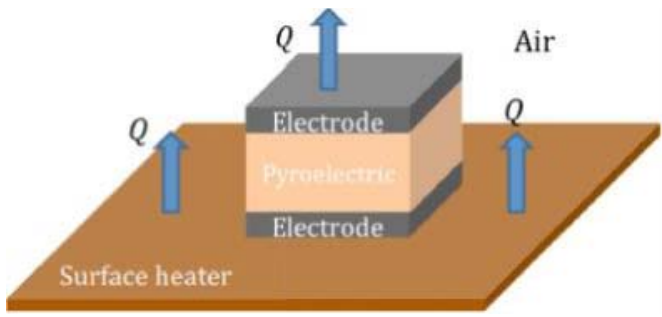

Fig. 2. Pyroelectric sensor on the surface configuration. Heat flows through the pyroelectric element along the polar axis.

for entropy flux measurement through the surface (as shown in Fig. 2). In the volume case, heat is transferred to the sensor from every direction, such that it is possible to measure entropy generation in the medium. In the case of surface sensors (to measure entropy flow through a surface), heat is conducted from one electrode plate to the other through a pyroelectric capacitor. In this case, the entropy flow is generated in the medium and transferred to the environment. In both cases, the sensor's temperature rises with heat absorption.

\section{B. Pyroelectric Response and Entropy Determination}

A pyroelectric material is a dielectric with polar point symmetry, which shows a spontaneous electrical polarization as a function of temperature. For pyroelectric flat-plate capacitors poled along the axis perpendicular to the plates, we assume a homogeneous pyroelectric material whose temperature, $T$, is uniform at any point in time; therefore, the generated pyroelectric current, $I$, is given by

$$
I=A \lambda \frac{d T}{d t}
$$

where $A$ is the electrode surface area of the material sample and $\lambda$ is the pyroelectric coefficient [22]. Integrating over time, the generated charge, $\Theta$, is

$$
\Theta=A \lambda\left(T_{f}-T_{i}\right)
$$

$T_{\mathrm{f}}-T_{\mathrm{i}}$ is the sensor temperature difference between the initial, $T_{\mathrm{i}}$, and final, $T_{\mathrm{f}}$, temperatures. From these expressions, temperature evolution can be directly inferred as

$$
\Delta T=T_{f}-T_{i}=\int \frac{I}{A \lambda} d t=\frac{\Theta}{A \lambda} .
$$

In addition, rearranging (3) we can write

$$
I=A \lambda \frac{d T}{d t}=\frac{A \lambda}{C_{\mathrm{p}}} \frac{d Q}{d t},
$$

where we consider a pure pyroelectric material with heat capacity $C_{\mathrm{p}}=\mathrm{d} Q / \mathrm{d} T$. If we assume the hypothesis of local equilibrium, entropy is defined locally and instantaneously [23]-i.e., if the pyroelectric sensor is at a particular temperature, $T$, at a particular time, $t$, then the entropy variation will be due to the heat transferred to the sensor, $\Delta Q$, as defined by (1). If this process is repeated in time, as illustrated in Fig. 3, then the total entropy variation will consist of the integral of the time intervals' variations if they are made short enough for quasistatic equilibrium. 


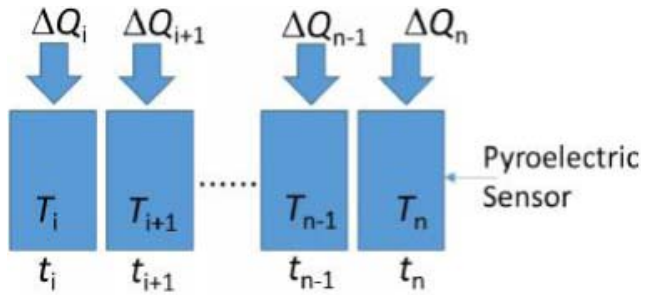

Fig. 3. Pyroelectric sensor that evolves from $T_{\mathrm{i}}$ at $t_{\mathrm{i}}$ to $T_{\mathrm{f}}$ at $t_{\mathrm{f}}$ while receiving $\Delta \mathrm{Q}$ heat over each interval, as inferred from [2].

With this approach, we can write, the entropy rate in the pyroelectric sensor for any particular interval at temperature $T$ as

$$
S_{\text {rate }}=\frac{d S}{d t}=\frac{C_{\mathrm{p}}}{A \lambda} \frac{I}{T} .
$$

If the generated current is integrated over time, entropy variation can be written as

$$
\Delta S=\int S_{\text {rate }} d t=\int \frac{C_{\mathrm{p}}}{A \lambda} \frac{I}{T} d t .
$$

Notice that $T$ is assumed to be constant over the examined intervals. While $S_{\text {rate }}$ is obtained from direct measurements of $I$ and $T, \Delta S$ can be found by numerically integrating $S_{\text {rate }}$.

If we substitute (3) into (8), we recover the well-known thermodynamic expression

$$
\Delta S=\int \frac{\delta Q}{T}=\int_{T_{\mathrm{i}}}^{T_{\mathrm{f}}} \frac{C_{p} \mathrm{~d} T}{T}=C_{\mathrm{p}} \ln \left(\frac{T_{\mathrm{f}}}{T_{\mathrm{i}}}\right),
$$

which is used in the discussion once the experimental results allow for numerical integration.

In summary, we have considered entropy and entropy rate variations in the pyroelectric sensor as functions of its parameters. Thus, both $\Delta S$ and $S_{\text {rate }}$ can be inferred with the pyroelectric sensor.

\section{Materials AND Methods}

Pyroelectric cells using two different materials with varying geometries are used. The two basic types were the commercial Polyvinylidene fluoride (PVDF) sensors depicted in Fig.4a and fabricated Lead zirconate titanate (PZT) cells. The PVDF sensors were provided by Measurement Specialties, Inc. [24]. They consist of PVDF deposited on a flexible plastic substrate. The PZT samples were fabricated using thick film technology. These cells consist of a layer of $4 \mathrm{~cm} \times 4 \mathrm{~cm}$ pyroelectric powder (Piezokeramica 856) sandwiched between two PdAg electrodes. Further details can be found elsewhere [25]. Cell details are described in Table 1, and the characteristics of the investigated materials are described in Table 2.

Pyroelectric sensors are usually modeled as current sources [26], such that the output current can be measured using a transresistance amplifier. The electrical circuit is depicted in Fig.5. The amplifier we use is an op-amp (TL074), and the feedback resistance, $R_{\mathrm{f}}$, is $1 \mathrm{M} \Omega$. The amplifier output voltage is measured using a computer-controlled data-acquisition system (either Agilent 34970A or

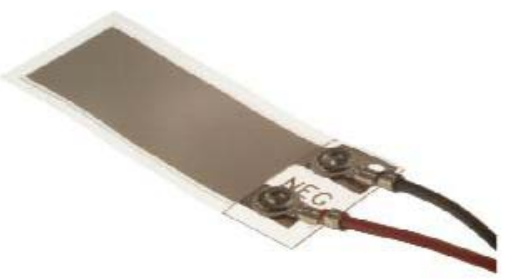

a)

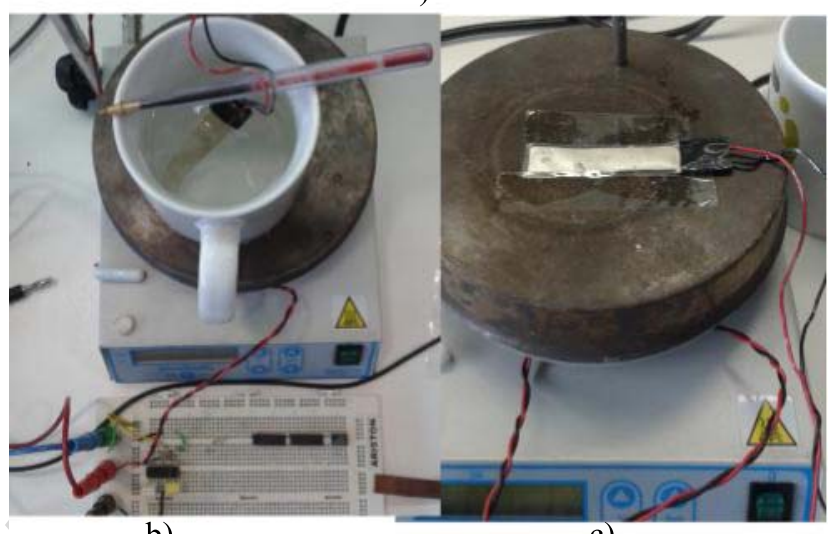

c)

Fig. 4. a) Pyroelectric film from Measurement Specialties. The sensor works as a temperature and heat sensor. b) Sensor inside the glycerine (volume measurement. c) Sensor on a heating surface (surface measurement).

TABLE I

GeOMETRIC PARAMETERS OF THE INVESTIGATED PYRoELECTRIC Sensors. Further Details Can Be Found in [13]

\begin{tabular}{|c|c|c|c|c|c|}
\hline & $\begin{array}{c}\mathrm{x} \\
(\mathrm{cm})\end{array}$ & $\begin{array}{c}\mathrm{y} \\
(\mathrm{cm})\end{array}$ & $\begin{array}{c}\mathrm{z} \\
(\mu \mathrm{m})\end{array}$ & $\begin{array}{c}\text { Mass } \\
\left(10^{-5} \mathrm{~kg}\right)\end{array}$ & $\begin{array}{c}\text { Pyroelectric } \\
\text { coefficient } \lambda \\
\left(10^{-6} \mathrm{Cm}^{-2} \mathrm{~K}^{-1}\right)\end{array}$ \\
\hline PVDF1 & 17.1 & 1.2 & 28 & 10.22 & 30 \\
\hline PVDF2 & 6.20 & 1.20 & 28 & 3.71 & 30 \\
\hline PZT2 & 4.00 & 4.00 & 60 & 48.0 & 178 \\
\hline PZT4 & 4.00 & 4.00 & 100 & 80.0 & 240 \\
\hline
\end{tabular}

TABLE II

Thermal Characteristics of the InVestigated Materials. THIS DATA IS USED FOR THE NUMERICAL SimUlations

\begin{tabular}{|c|c|c|c|}
\hline Material & $\begin{array}{c}\text { Specific } \\
\text { heat capacity } \\
c_{\mathrm{p}}\left(\mathrm{J} \mathrm{kg}^{-1} \mathrm{~K}^{-1}\right)\end{array}$ & $\begin{array}{c}\text { Thermal } \\
\text { conductivity } \\
\left(\mathrm{W} \mathrm{m}^{-1} \mathrm{~K}^{-1}\right)\end{array}$ & $\begin{array}{c}\text { Density } \\
\left(\mathrm{kg} \mathrm{m}^{-3}\right)\end{array}$ \\
\hline $\begin{array}{c}\text { Electrode } \\
(\mathrm{Ag} / \mathrm{Pd} 2.8: 1)\end{array}$ & 241 & 318 & 10890 \\
\hline PZT & 440 & 1.25 & 7500 \\
\hline PVDF & 962 & 23 & 1780 \\
\hline Alumina & 880 & 0.289 & 3750 \\
\hline Glycerol & 2424 & & 1261 \\
\hline
\end{tabular}

Yokogawa DL750). The relation between the input current 201 and the output voltage is given by

$$
I=-\frac{V_{0}}{R_{\mathrm{F}}}
$$

To determine the experimental entropy variation, we conduct two different types of experiments: one measuring heat source entropy generation and the other measuring heat flow through the source's surface. In the first one, a $250-\mathrm{ml}$ 


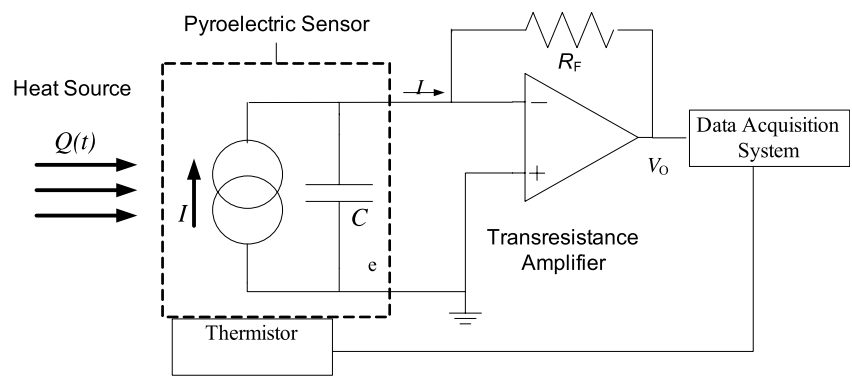

Fig. 5. Equivalent pyroelectric sensor model connected to a transresistance amplifier. The thermistor is placed so as to settle the initial temperature of the pyroelectric sensor in the environment while the pyroelectric temperature monitoring is carried out directly based on its current profile [15].

glycerin thermal bath at $343 \mathrm{~K}\left(70{ }^{\circ} \mathrm{C}\right)$ is prepared using a heater Ovan - MicroMagmix as the heat source. The samples, originally at room temperature, are dipped into the bath. Glycerin is used because it is a good electrical insulator and its boiling point is $563 \mathrm{~K}$ (Fig. 4b). In the second experiment, we attach the pyroelectric sensor to a metallic surface that acts as a heat source whose temperature is set at $343 \mathrm{~K}$, as illustrated by the surface heater in Fig. 4c.

The physical structures of the pyroelectric film (see Fig. 4, schematically illustrated in Figs 1-2) with the data described in Table 1 are modeled using finite-element software (Comsol Multiphysics). One-dimensional (1D) thermal simulations are performed using the data in Table 1, and, taking advantage of sample symmetries along the z-axis, they can be extrapolated to 3D. Border effects are neglected, which is a common approximation for planar capacitors. Moreover, metal electrodes are better thermal conductors than dielectric materials; therefore, they do not limit heat transfer from the heat source to the pyroelectric material. Thermal simulations consider a heat source at constant temperature (a glycerin bath at the experimental temperature), with the pyroelectric sensor being dipped at an initial temperature equal to the ambient temperature. Thus, temperature, enthalpy, and entropy evolutions are monitored in the capacitor structure with consideration of the metallic electrodes. The electrical response of the pyroelectric sensor is not simulated. Similarly, the experimental setup for the surface measurement illustrated in Fig. $4 \mathrm{c}$ is modeled considering a surface temperature source and air as the environment. Convection is not considered.

\section{Results}

In this section, we present the experimental results for the electrical responses of the different pyroelectric sensors when exposed to various heating conditions. These results are compared with simulation analyses.

\section{A. Heat Measurements in Dipped Samples}

Heat is related to pyroelectric current by (3) using the $C_{\mathrm{p}}$-value for the sensor. Enthalpy, $H$, is the parameter investigated in simulations $(H=U+P \cdot V$, where $U$ is the internal energy, $P$ is the pressure, and $V$ is the volume). If no external work is done on the solids, the enthalpy equals heat variation, which is the case for these experiments. The comparison

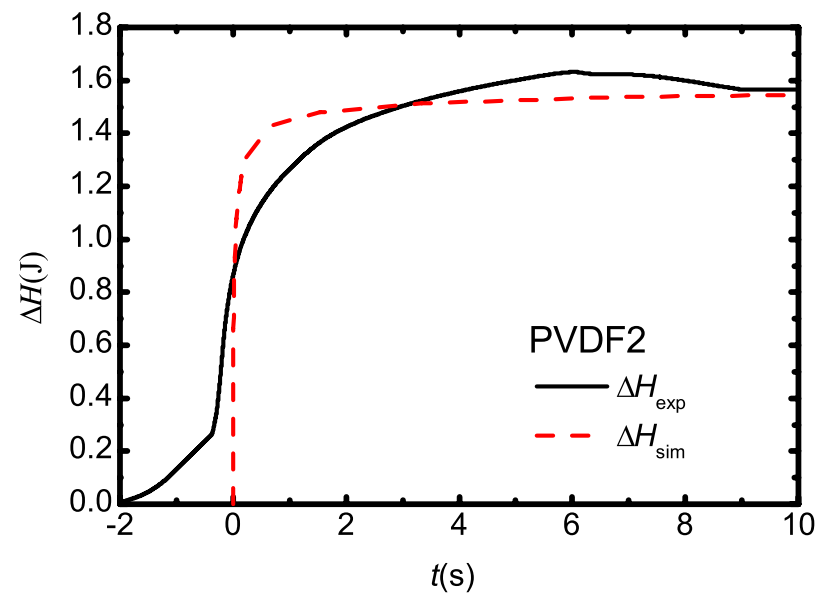

Fig. 6. Enthalpy variation in the PVDF2 sensor for a temperature change of $45 \mathrm{~K}$. Comparison between experimental results and simulation shows good agreement.

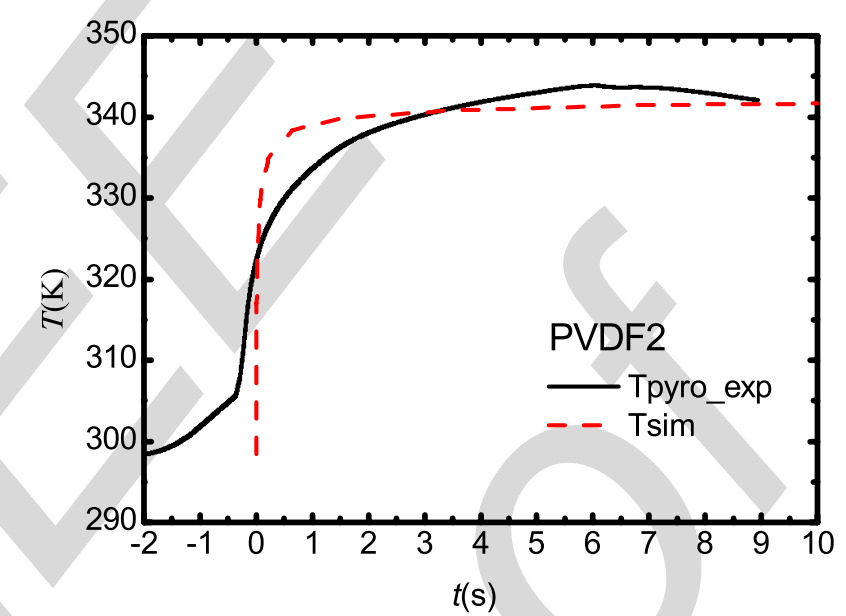

Fig. 7. Experimental and simulated temperature profiles in the PVDF2 sensor obtained using (5).

between the experimental results and the simulation curve is presented in Fig. 6. There is good agreement, though slight differences are found in the transient dynamic process; these differences arise from the practical setup of dipping the sample in the glycerin bath.

\section{B. Temperature Measurement in Dipped Samples}

The temperature in the pyroelectric sensor is inferred from (5). Experimental results for the PVDF2 sample compared with the pyroelectric temperature obtained from the simulation based on the heat transfer equation are presented in Fig. 7. The pyroelectric sensor follows the expected temperature variation as described in thermometry references [16], [18]. This avoids the need for external temperature sensors with different time constants, which cannot measure the pyroelectric's inner temperature.

\section{Entropy Determination in Dipped Samples}

Entropy is immediately obtained from heat and temperature using (9). The experimental results are compared with the simulation curves, and there is good agreement (see Fig. 8). 


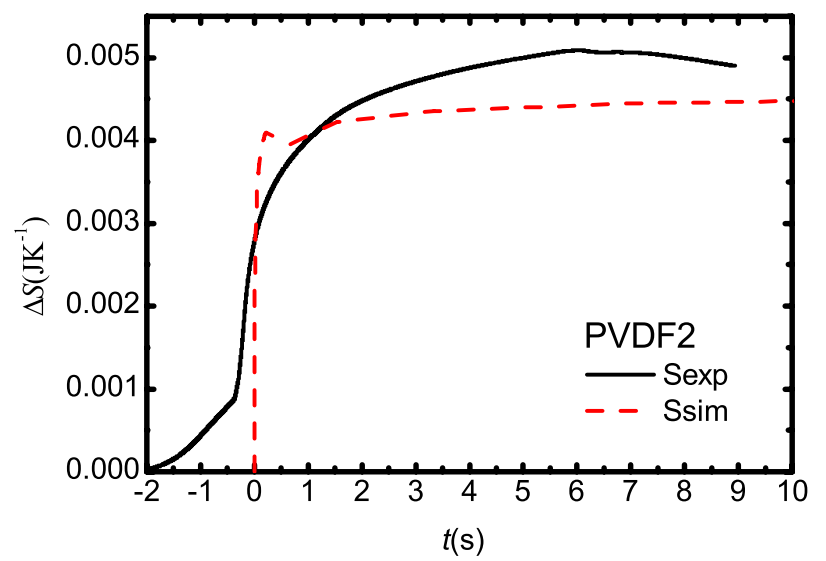

Fig. 8. Experimental and simulated entropy-generation in the PVDF2 sensor.

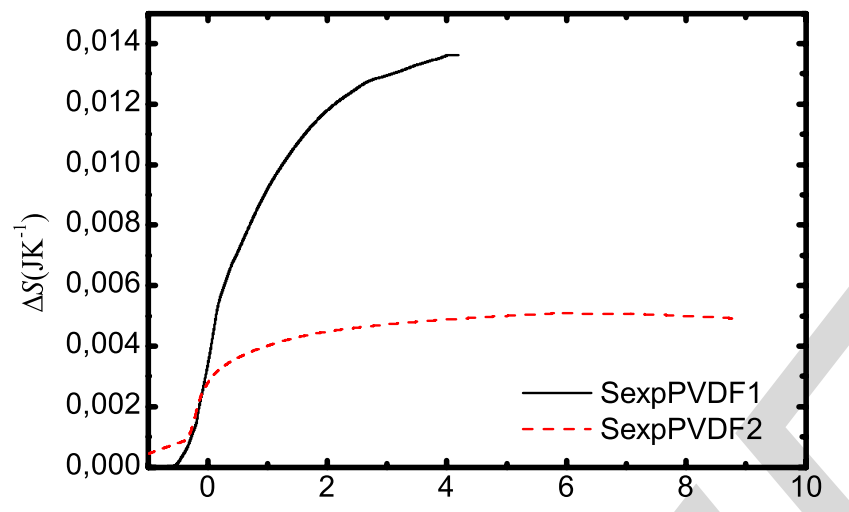

$t(\mathrm{~s})$

Fig. 9. Measured entropy variation for PVDF sensors with two different volumes.

\section{Geometry Comparison in Dipped Samples}

Entropy is an extensive property; thus, it scales with volume. We compare the entropy generation of two PVDF sensors of various sizes (PVDF1 and PVDF2 in Table 1). Their volume ratio is 2.76 . Their experimental entropy ratio after heating is approximately 2.7 , which perfectly matches the volume ratio, as illustrated in Fig. 9.

\section{E. Surface Measurements}

The relationship between the entropy flux and volume measurements is investigated according to the setups described in Figs. 1 and 2. The expected differences are found at the maximum entropy change (see Fig. 10). Experimental results illustrated in Fig.11 show good agreement with the simulation for the total $\Delta S$ but larger differences during the dynamic response of the sensor. These deviations are discussed later.

\section{Discussions}

\section{A. Model and Approximations}

In this section, we first discuss the impact of the assumptions used in heat transfer equation (2); later, we evaluate the experimental results according to thermodynamics and simulation.

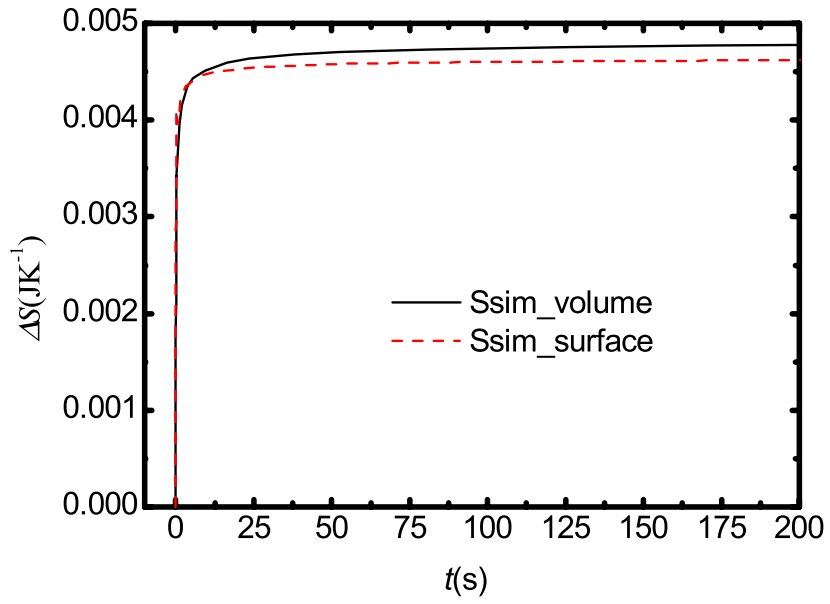

Fig. 10. Simulations of entropy generation in the pyroelectric sensor PVDF2 for two cases: 1) surface measurement, where one side of the sensor is at room temperature because of contact with air (red dashed line) and 2) volume measurement, where the sensor is completely inside the glycerin bath (black solid line).

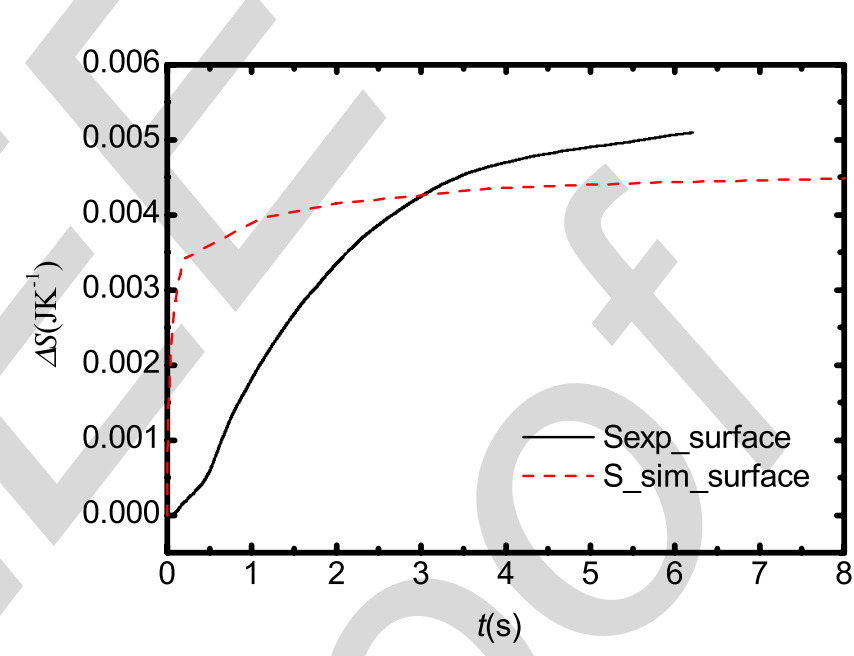

Fig. 11. Comparison between experimental and simulated entropy flux for the surface measurements.

Heat convection may have had an effect on the pyroelectric measurements because the samples were dipped into the bath. To evaluate this effect, we evaluate Biot's number, defined as $\mathrm{Bi}=h \cdot L / k$, where $h$ is the convective parameter (estimated as approximately 15), $k$ is the thermal conduction, and $L$ is the thickness of the sample [20]. In our case, $\mathrm{Bi}=0.05$. As long as $\mathrm{Bi}<0.1$, thermal conduction is fast enough to minimize thermal gradients; thus, convection is negligible.

In heat transfer equation (2), heat is transferred conductively in accordance with $k \cdot \nabla^{2} T$. The temperature gradient is found to be linear inside the pyroelectric sensor, both for the volume and surface simulations. Thus, the second-order temperature derivative is zero, and this term does not contribute. Consequently, time-dependent heat transfer occurs, which is convenient for pyroelectric conversion and entropy estimation, as inferred from the comparison of (2) and (3). However, pyroelectric equation (3) assumes that temperature is homogenous inside the pyroelectric, whereas 


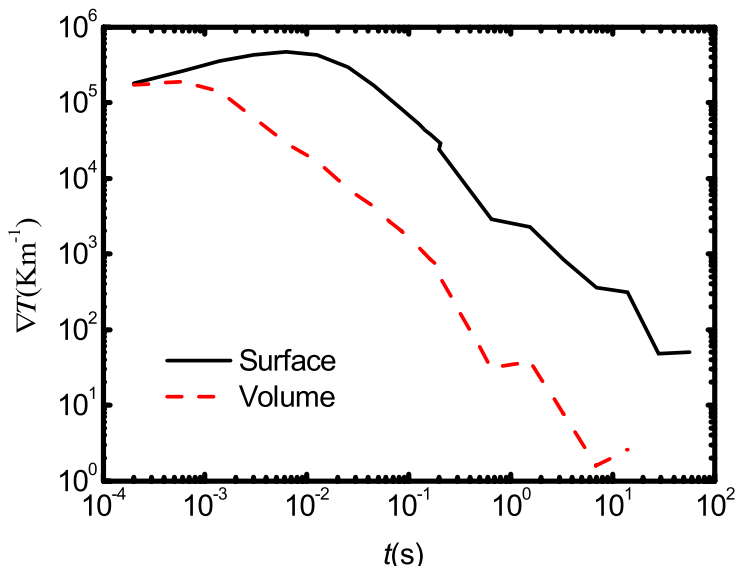

Fig. 12. Simulation of the thermal gradient magnitude-evolution inside the sensor for volume and surface measurements.

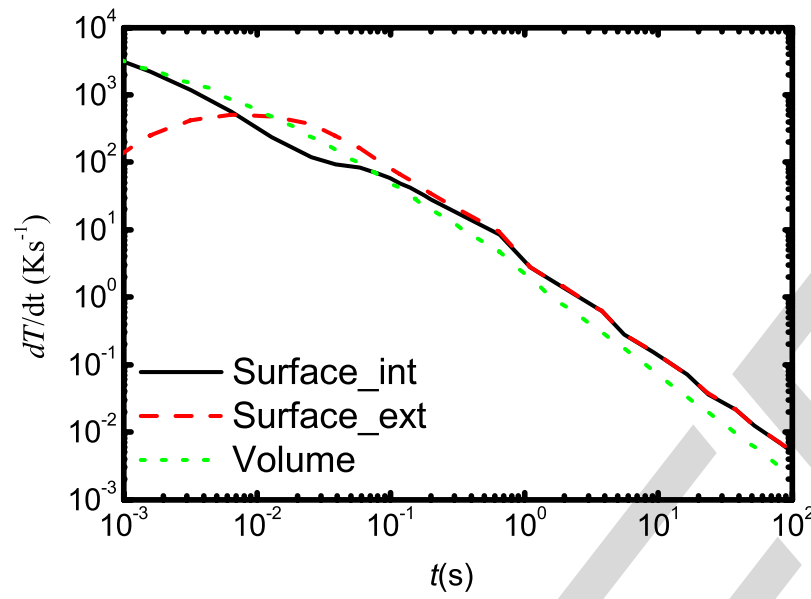

Fig. 13. Simulated temperature derivative, dT/dt, for the surface measurement at the hot side (surface_int), at the cold side (surface_ext), and for the volume measurement (volume). the gradient is time-dependent, as illustrated in Fig. 12. In the model described in Section II, the sensor is approximated as a lumped system; that is, during transient heat transfer, the temperature does not vary with position because of the small dimensions and high conductivity terms and small gradients, but the system temperature varies uniformly with time. This approximation is found to be valid for times greater than $0.1 \mathrm{~s}$ after the initial dipping; however, in the initial transient heat transfer, temperatures must be homogenized, as obtained from simulations and illustrated in Fig.13, where the temperatures at both surfaces of the pyroelectric and the average temperature converge. Larger differences should be observed for surface measurements, which explain the transient differences between simulations and experimental results in Fig.11. For this case, improved analyses of the pyroelectric coefficient, $\lambda$, as a function of position may improve agreement. Finally, we assume that both thermal capacity, $C_{\mathrm{p}}$, and the pyroelectric coefficients, $\lambda$, are temperature-independent in this first study. This is a reasonable approximation, considering the good agreement between the thermodynamic model and the experimental results. Future studies attempting to characterize $C_{\mathrm{p}}(T)$ will be necessary to take this second-order effect into account.

\section{B. Results and Thermodynamics Model}

Results comparing sensor geometries show that we can characterize entropy generation, which is an extensive parameter, if volume is normalized and thus becomes independent of the sensor geometry. Thus, whatever the sensor geometry is, realistic values can be achieved in environmental measurements, though the sensor should be as small as possible in order to not affect the environmental temperature. In our experiments, the environment is the thermal bath heat source. Good agreement between volume and surface measurements was found, although the transient evolution model should be improved for better agreement in the transient regions (Fig. 11). Surface measurements may be improved using a specific sensor for entropy measurements, thereby minimizing the transient discrepancy.

Entropy-generation in the PVDF sample increases when dipped in the thermal source, in accordance with (9), as illustrated in Fig. 8 and compared in Table 2. The real interest of this work, however, is to relate the sensors' entropy to environmental entropy generation; thus, it is convenient to validate these results using thermodynamic models. We can estimate the theoretical thermodynamic entropy for the thermal bath from (11) and (12). While the samples reach the bath temperature, the bath, which is considered to bea heat source, undergoes a change $\Delta T<0.2 \mathrm{~K}$.

$$
\Delta S=\frac{\Delta Q}{T}=\frac{C_{p} \Delta T}{T}=\frac{C_{\mathrm{p}} \cdot\left(T_{\mathrm{f}}-T_{\mathrm{i}}\right)}{T_{\mathrm{i}}},
$$

where

$$
T_{\mathrm{f}}=\frac{\sum_{i} C_{\mathrm{p}, \mathrm{i}} \cdot T_{\mathrm{i}}}{\sum_{\mathrm{i}} C_{\mathrm{p}, \mathrm{i}}},
$$

and the sum is calculated for all of the elements involved in the problem (heat source and sensors in this case). The pyroelectric sensor's temperature is not constant; thus, the entropy variation is given by

$$
\Delta S=\int \frac{\delta Q}{T}=\int_{T_{\mathrm{i}}}^{T_{\mathrm{f}}} \frac{C_{p} \mathrm{~d} T}{T}=C_{\mathrm{p}} \ln \left(\frac{T_{\mathrm{f}}}{T_{\mathrm{i}}}\right),
$$

which is compared with the experimental data obtained through numerical integration of the experimental results using

$$
\begin{aligned}
\Delta S & =\int \frac{C_{\mathrm{p}}}{A \lambda} \frac{I}{T} d t=\int \frac{C_{\mathrm{p}}}{A \lambda} \frac{I}{\left(T_{i}+\int \frac{I}{A \lambda} d t\right)} d t \\
& \approx \frac{C_{\mathrm{p}}}{A \lambda T_{\mathrm{i}}} \int I\left(1-\frac{\int \frac{I}{A \lambda} d t}{T_{\mathrm{i}}}\right) d t \approx \frac{C_{\mathrm{p}} \Theta}{A \lambda T_{i}}\left(1-\frac{\frac{\Theta}{A \lambda}}{T_{i}}\right),
\end{aligned}
$$

as inferred from (5) and (9).

The comparison of the predictions of this thermodynamic model with the experimental results achieved with equations $7-14$ for the investigated samples is shown in Table 3. To compare different materials, we take the data for 
TABLE III

COMPARISON BETWEEN DyNAMiC SimUlations, THERMOdynamic Models and EXPerimental Results in Volume (GLyCERINE) AND SuRface (AIR) MEASUREMENTS

\begin{tabular}{|l|l|l|l|l|l|l|}
\hline & & & \multicolumn{2}{|c|}{$\begin{array}{c}\text { Thermodynami } \\
\text { cs model }\end{array}$} & \multicolumn{2}{|c|}{ Experimental } \\
\hline & $\begin{array}{l}\text { Heat } \\
\text { Sourc } \\
\text { e }\end{array}$ & $\begin{array}{l}\Delta \mathrm{T} \\
(\mathrm{K})\end{array}$ & $\begin{array}{l}\mathrm{H} \\
(\mathrm{J})\end{array}$ & $\begin{array}{l}\Delta \mathrm{S} \\
\left(\mathrm{JK}^{-1}\right)\end{array}$ & $\mathrm{H}(\mathrm{J})$ & $\begin{array}{l}\Delta \mathrm{S}^{-}\left(\mathrm{JK}^{-1}\right. \\
)\end{array}$ \\
\hline PVDF1 & $\begin{array}{l}\text { Glyce } \\
\text { rin }\end{array}$ & 45 & 4.42 & 0.0138 & 4.36 & 0.0133 \\
\hline PVDF2 & $\begin{array}{l}\text { Glyce } \\
\text { rin }\end{array}$ & 45 & 1.60 & 0.00501 & 1.58 & 0.00504 \\
\hline PVDF1[25] & Air & 68 & 2.85 & 0.0202 & 2.90 & 0.0203 \\
\hline PVDF2[25] & Air & 80 & 6.69 & 0.00848 & 6.94 & 0.00832 \\
\hline PZT1[25] & Air & 30 & 15.23 & 0.049 & 12.91 & 0.040 \\
\hline PZT4[25] & Air & 28 & 15.23 & 0.049 & 15.4 & 0.049 \\
\hline
\end{tabular}

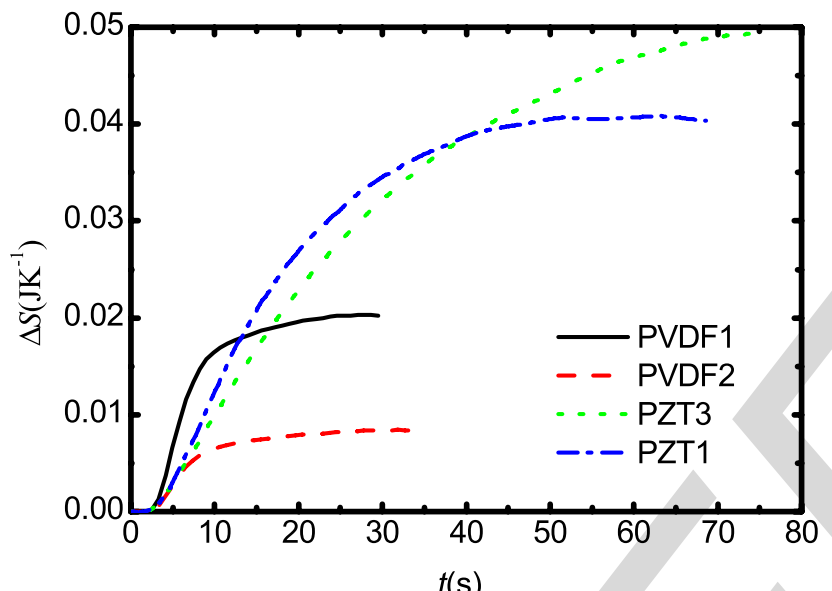

$t(\mathrm{~s})$

Fig. 14. Comparison between entropy generation in PVDF and PZT heated with air flow in accordance with data from [25]. As illustrated in Table 3 , experimental results agree with the thermodynamic model, regardless of the material used (PVDF vs. PZT) or the heating source (air flow vs. glycerin bath).

PZT from [25]. In that case, samples were heated with air flow; the results are illustrated in Fig. 14. The comparison between the obtained enthalpies and entropies for different temperature excursions also shows good agreement with experimental results, as depicted in Table 3. These results are also independent of the type of pyroelectric sensor (PVDF vs. PZT) and even the heat source (glycerin bath vs. air flow), demonstrating the effective behavior of the entropy sensor.

\section{Beyond the Sensor Entropy Measurement: The Environmental Measurement}

Up to this point, we have discussed the entropy accumulated in the pyroelectric sensor. The interesting parameter, however, is the entropy released by the heat source, which is given by (11) and (12) for the case in which the $C_{\mathrm{p}}$ of the heat source is known. In our experimental setup for the glycerin bath, $\Delta S_{\mathrm{hs}}=-4.68 \cdot 10^{-3} \mathrm{~J} / \mathrm{K}$. The difference between the entropy released by the heat source, $\Delta S_{\mathrm{hs}}$, and that absorbed by the sensor, $\Delta S_{\text {sensor }}$ (PVDF2 sample), is the entropy gained by the Universe, $\Delta S_{\mathrm{uni}}=\Delta S_{\mathrm{hs}}-\Delta S_{\text {sensor }}$. According to the second principle of thermodynamics, $\Delta S_{\text {uni }}>0$ for irreversible processes, and $\Delta S_{\text {uni }}=0$ for reversible processes. We find that $\Delta S_{\text {uni }}=8.62 \cdot 10^{-3} \mathrm{~J} / \mathrm{K}$ and $0.37 \cdot 10^{-3} \mathrm{~J} / \mathrm{K}$ for PVDF1 and PVDF2, respectively. Though $\Delta \mathrm{S}_{\text {uni }}>0$ in both cases, indicating that they are irreversible, the overall measurement accuracy must be improved for exact heat source entropy determination. This result is of particular interest for various reasons. First, because the size of the sensor is related to entropy generation, it should be kept small (note that $\Delta S_{\text {uni }}$ is larger for PVDF1 than for PVDF2). Second, in the glycerin bath, a simple thermodynamic model is easily obtained and comparison between $\Delta S$ for the sensor and heat source is straightforward; however, in the case of air flow, the model is much more complex. However, the entropy generation at the sensor is a good estimation for both heating sources, i.e., the thermal bath and the surface heater. In fact, for PVDF2, the sensor entropy is $5.04 \cdot 10^{-3} \mathrm{~J} / \mathrm{K}$ and the generated entropy is $\Delta S_{\text {uni }}=0.37 \cdot 10^{-3} \mathrm{~J} / \mathrm{K}$. The difference between the sensor and the heat source entropy is $\sim 7.3 \%$, which could be reduced even more by scaling down the sensor size, with a limit imposed by the second principle. Also, we have kept a surface at room temperature. This sensor could be placed in an isolated environment to improve measurements.

\section{CONCLUSION}

We have investigated pyroelectric materials as entropy sensors and proved the concept from these results. Heat and temperature are estimated, and, thus, entropy is inferred. Geometry-dependence did not affect normalized entropy determination, as was theoretically expected.

Both volume entropy and entropy flow were measured. Whereas volume measurements showed good agreement with theoretical estimations, surface measurements only provided a good estimation of the total entropy flow, and transient entropy evolution should be investigated in more detail. Finally, the changes in the heat source and universe entropy could be obtained with our sensor using convenient thermodynamic models, which should provide insights into system energy yield.

Our accuracy was around $10 \%$, which is encouraging for this initial stage of testing, because the sensor was able to predict the general trend even though the system has not yet been optimized. Possible avenues to improve these sensors include designing a pyroelectric sensor that minimizes geometrical dimensions and substrate effects with improved thermal conduction while maintaining a small thermal capacity.

These sensors have possible applications in characterization of both electrochemical processes and irreversible thermodynamic processes, like combustion, heat engines, and electrical systems with Joule dissipation.

\section{REFERENCES}

[1] A. Navid, D. Vanderpool, A. Bah, and L. Pilon, "Towards optimization of a pyroelectric energy converter for harvesting waste heat," Int. J. Heat Mass Transf., vol. 53, nos. 19-20, pp. 4060-4070, Sep. 2010.

[2] A. Bejan, Advanced Engineering Thermodynamics, 2nd ed. Hoboken, NJ, USA: Wiley, 2006.

[3] M. W. Zemansky and R. H. Dittman, Heat and Thermodynamics. New York, NY, USA: McGraw-Hill, 1990. 
[4] C.-J. Ding and L.-F. Luo, "Experimental study of entropy production in cells under alternating electric field," Chin. Phys. Lett., vol. 29, no. 8 , p. 088701, Aug. 2012.

[5] V. Basso, F. Russo, J.-F. Gerard, and S. Pruvost, "Direct measurement of the electrocaloric effect in poly(vinylidene fluoride-trifluoroethylenechlorotrifluoroethylene) terpolymer films," Appl. Phys. Lett., vol. 103, no. 20, p. 202904, 2013.

[6] X. Yuan and F. Yang, "Energy transfer in pyroelectric material," in Heat Conduction-Basic Research, V. S. Vikhrenko, Ed. Beijing, China: InTech, 2011, pp. 229-248.

[7] Z. Jiang, J. Zhang, L. Dong, and J. Zhuang, "Determination of the entropy change of the electrode reaction by an ac electrochemicalthermal method," J. Electroanal. Chem., vol. 469, no. 1, pp. 1-10, Jun. 1999.

[8] M. Mansour, N. Chokani, A. I. Kalfas, and R. S. Abhari, "Time-resolved entropy measurements using a fast response entropy probe," Meas. Sci. Technol., vol. 19, no. 11, p. 115401, Nov. 2008.

[9] A. Eddahech, O. Briat, and J.-M. Vinassa, "Lithium-ion battery heat generation investigation based on calorimetric entropy measurements," in Proc. IEEE Int. Symp. Ind. Electron., May 2013, pp. 1-5.

[10] Y. Troxler et al., "The effect of thermal gradients on the performance of lithium-ion batteries," J. Power Sour., vol. 247, pp. 1018-1025, Feb. 2014.

[11] R. A. Huggins, Energy Storage. New York, NY, USA: Springer, 2010.

[12] M. Naderi and M. M. Khonsari, "Thermodynamic analysis of fatigue failure in a composite laminate," Mech. Mater., vol. 46, pp. 113-122, Mar. 2012.

[13] A. Cuadras, V. J. Ovejas, and M. Quilez, "Entropy as a wear out indicator: The resistor example," in Proc. 10th Int. MultiConf. Syst., Signals Devices (SSD), Hammamet, Tunisia, 2013, pp. 1-5.

[14] V. J. Ovejas and A. Cuadras, "Battery state of charge determination from heat and entropy measurements," in Proc. 12th Int. Conf. Energy Storage, Lleida, Spain, 2012.

[15] A. Cuadras and V. J. Ovejas, "Pyroelectric sensor for entropy measurements," in Proc. IEEE 11th Int. Multi-Conf. Syst., Signals Devices (SSD), Feb. 2014, pp. 1-5.

[16] C. F. Tsai and M. S. Young, "Pyroelectric infrared sensor-based thermometer for monitoring indoor objects," Rev. Sci. Instrum., vol. 74, no. 12 , p. $5267,2003$.

[17] A. Rogalski, "Infrared detectors: Status and trends," Prog. Quantum Electron., vol. 27, nos. 2-3, pp. 59-210, 2003.

[18] P. R. N. Childs, J. R. Greenwood, and C. A. Long, "Review of temperature measurement," Rev. Sci. Instrum., vol. 71, no. 8, p. 2959, 2000.

[19] S. B. Lang and S. Muensit, "Review of some lesser-known applications of piezoelectric and pyroelectric polymers," Appl. Phys. A, Mater. Sci. Process., vol. 85, no. 2, pp. 125-134, Nov. 2006.

[20] A. F. Mills, Heat Transfer, 2nd ed. Upper Saddle River, NJ, USA: Prentice-Hall, 1998.
[21] S. B. Lang, "Pyroelectricity: From ancient curiosity to modern imaging tool," Phys. Today, vol. 58, no. 8, pp. 31-36, 2005.

[22] Y. Xu, Ferroelectric Materials and Their Applications. Amsterdam, The Netherlands: Elsevier, 1991.

[23] G. Lebon, D. Jou, and J. Casas-Vázquez, Understanding Nonequilibrium Thermodynamics: Foundations, Applications, Frontiers. New York, NY, USA: Springer, 2008.

[24] (Jul. 2014). Measurement Specialties. [Online]. Available: http://www.meas-spec.com/

[25] A. Cuadras, M. Gasulla, and V. Ferrari, "Thermal energy harvesting through pyroelectricity," Sens. Actuators A, Phys., vol. 158, no. 1, pp. 132-139, 2010.

[26] V. Ferrari, D. Marioli, and A. Taroni, "Displacement sensor based on pyroelectric thick films and contactless light-spot cursor," IEEE Trans. Instrum. Meas., vol. 51, no. 4, pp. 819-823, Aug. 2002.

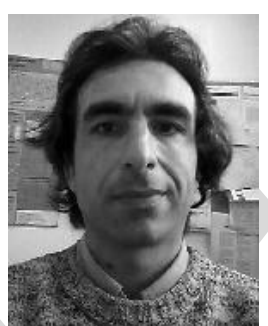

Angel Cuadras was born in Tarragona in 1974. $\mathrm{He}$ received the B.S., M.S., and Ph.D. degree in physics from the University of Barcelona, in 1997, 1999, and 2002, respectively. He was involved in the technology and growth of $\mathrm{Si}_{1-x-y} \mathrm{Ge}_{x} \mathrm{C}_{y}$ materials and the physical and electrical characterization of MOS capacitors. In 2003, he joined the Instrumentation, Sensors, and Interfaces Group, Universitat Politècnica de Catalunya, where he is currently an Associate Professor. His main interests are energy systems monitoring (with a major focus on battery state of health and state of charge) and characterization of systems using impedance spectroscopy and entropy characterization. He is a member of the IEEE Instrumentation and Measurement Society. He was the Chair of the IEEE 11th Multi-Conference on Signals, Systems, and Devices (2014).

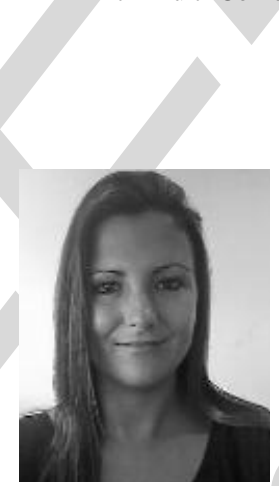

Victoria Julia Ovejas was born in Barcelona in 1985. She received the B.S. and M.S. degrees in telecommunications engineering from the Universitat Politècnica de Catalunya (UPC), in 2008 and 2010, respectively, where she is currently pursuing the Ph.D. degree in electronics engineering. She is a member of the Instrumentation, Sensors, and Interfaces Group with UPC. Her main interests are Li-ion battery monitoring (including state of charge and state of health) and energy harvesting-related subjects. 


\section{AUTHOR QUERIES}

AQ: 1 = Please check whether the edits made in the financial section are OK.

AQ:2 = Please provide the year of the IEEE sensors conference.

AQ:3 = Please provide the page range for ref. [14].

AQ:4 = Please note that references [18] and [27] are the same, hence we deleted Ref. [27]. This change will also reflect in the citations present in the body text. Please confirm.

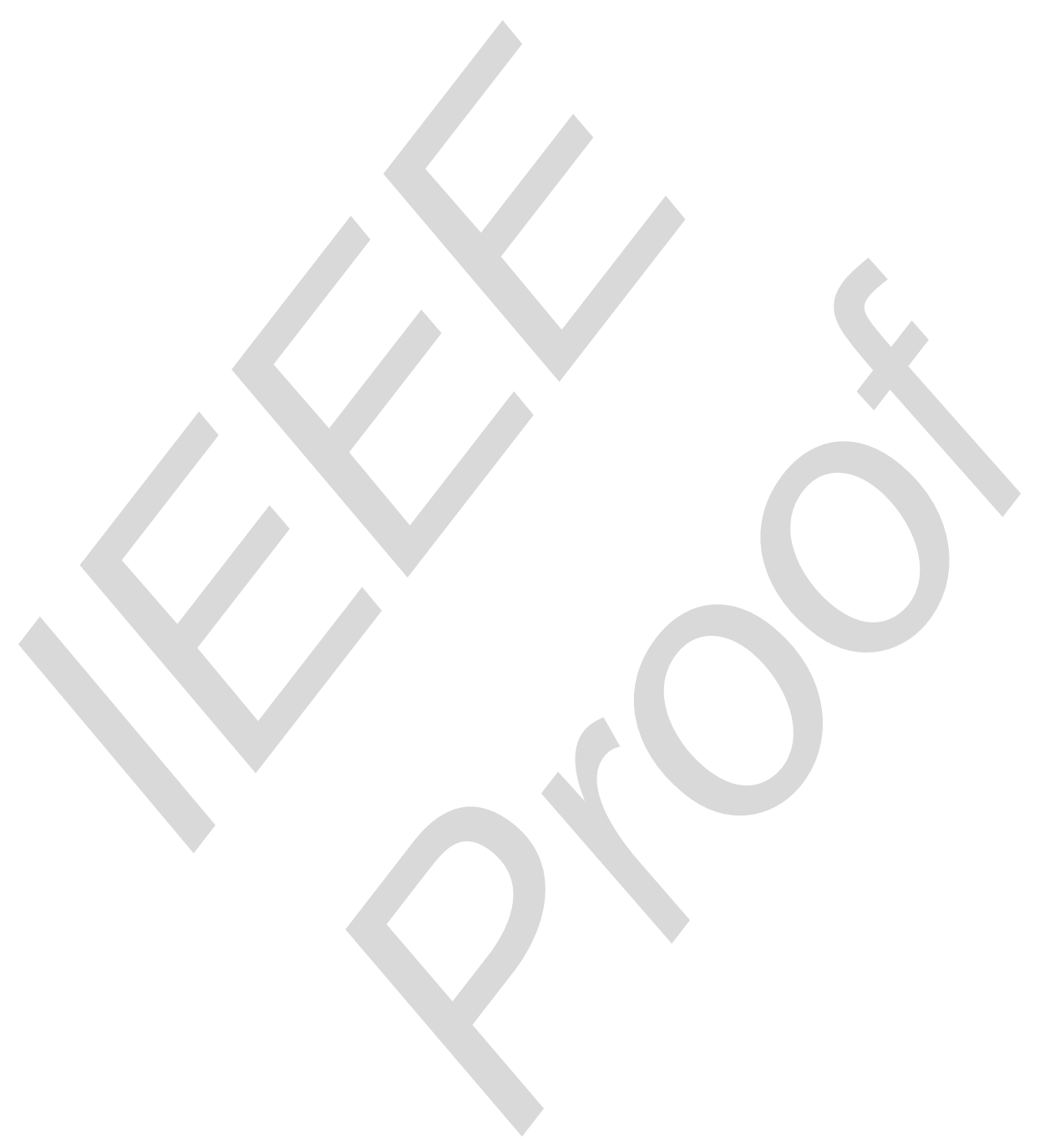




\title{
Determination of a System's Entropy Using Pyroelectric Sensors
}

\author{
Angel Cuadras, Member, IEEE, and Victoria Julia Ovejas
}

\begin{abstract}
We propose a system for measuring entropy variations, $\Delta S$, in thermal systems using pyroelectric sensors. These sensors convert time-dependent temperature variations into electrical current. Consequently, heat and temperature variations are obtained, and sensor entropy is inferred. Various polyvinylidene fluoride and lead zirconate titanate sensors have been tested. Two types of measurements are performed. One in the volume, to measure entropy variations in a heat source, and the other on the surface, to measure entropy flux delivered by the heat source. Thermodynamic models and heat transfer dynamic simulations agree with the experimental results and relate the sensor entropy to the heat source entropy. These results show that pyroelectric sensors can enable entropy monitoring of thermal processes to improve system performance.
\end{abstract}

Index Terms-Entropy, heat, pyroelectric sensor, temperature.

\section{INTRODUCTION}

I $\mathrm{N}$ ENERGY generation systems, output energy is the target to be maximized, whereas energy efficiency must retain the maximum yield. However, losses during energy conversion can reach up to $60 \%$, with most of it being dissipated as low-grade thermal energy [1]. For instance, in electrical energy generation, gas, wind, and water turbine efficiencies are as large as $40 \%, 60 \%$, and $90 \%$, respectively. The efficiency is around $30 \%-60 \%$ in electrical motors, $10 \%-50 \%$ in combustion engines, and $20 \%-50 \%$ in refrigerators. Consequently, energy conversion efficiency is a critical issue that must be addressed, i.e., the output energy for a given input energy should be maximized.

Energy loss is connected to entropy generation. Entropy is a common parameter in chemical reactions and is commonly studied in thermodynamics [2], [3], biology [4], and materials science [5]-[7]. From a technical viewpoint, entropy is considered in the design of refrigeration and combustion engines for thermodynamic efficiency [2], [8] as well as in batteries [9], [10]. However, entropy generation in irreversible

Manuscript received June 16, 2015; revised July 30, 2015; accepted August 3, 2015. This work was supported by the Ministerio de Ciencia e Innovación through the Spanish Government under Contract TEC2011-27397. The work of V. J. Ovejas was supported by the Ministerio de Educación, Cultura y Deporte through the Formación del Profesorado Universitario. This is an expanded paper from the IEEE SENSORS 200X Conference. The associate editor coordinating the review of this paper and approving it for publication was Prof. Zeynep Celik-Butler.

The authors are with the Department of Electronic Engineering, Escola d'Enginyeria de Telecomunicació i Aeronàutica de Castelldefels, Universitat Politècnica de Catalunya, Barcelona 08034, Spain (e-mail: angel.cuadras@upc.edu; victoria.julia.ovejas@upc.edu).

Color versions of one or more of the figures in this paper are available online at http://ieeexplore.iee.org.

Digital Object Identifier 10.1109/JSEN.2015.2466112 processes is not commonly monitored in real time, although it can be interesting to do so in many fields ranging from thermal energy management to aging systems [2], [11], [12]. In our previous research [13], [14], we found that entropy can be used to estimate the failure and aging of an electrical circuit because it increases monotonously; thus, a threshold for maximum tolerated wear out, either for electrical or mechanical systems, can be defined.

Heat and temperature measurements are needed to estimate entropy. Heat is usually measured using calorimeters, which are not practical for in situ measurements. Heat flux sensors based on the Seebeck effect are also being used. With respect to temperature, common sensors are thermistors, thermocouples, RTDs, and pyroelectric sensors. Thermistors and RTDs are semiconductors and conductors, respectively, whose resistances are temperature dependent. Thermocouples are based on the Seebeck effect, and pyroelectric sensors are sensitive to time-dependent temperature fluctuations. Our approach is to relate temperature and heat in the sensor through the thermal capacity, $C_{\mathrm{p}}=\mathrm{dQ} / \mathrm{dT}$. Among the possible sensors, pyroelectric sensors are preferred because they are bulk sensors with a well-defined heat capacity and need no external bias. Thermocouples were discarded because they are junctions of two materials, and RTDs and thermistors need to be biased. Pyroelectric sensors convert temperature variations into electrical currents; they have been widely commercialized as presence detectors, fire detectors, and infrared detectors [16]-[19].

In this work, we investigate the use of pyroelectric sensors in the monitoring of temperature fluctuations, obtain the heat involved in the process via heat capacity, and estimate the sensor entropy variation. We consider entropy measurements in volumes and entropy flux through surfaces (a preliminary study was presented in [15]). Sensor entropy is finally related to entropy change in the system under study using a convenient model. This paper is organized as follows. In Section II, a phenomenological model describing the relationship between the basic heat conduction equation, thermodynamics, and pyroelectric current generation is described. In Section III, we describe the materials and methods used to test our hypothesis. In Section IV, we report on the entropy measured using various pyroelectric sensors; finally, we discuss our results and summarize the conclusions.

\section{Theoretical ApProACH}

The aim of this research is to measure entropy variation in a heat source using pyroelectric sensors. These sensors 


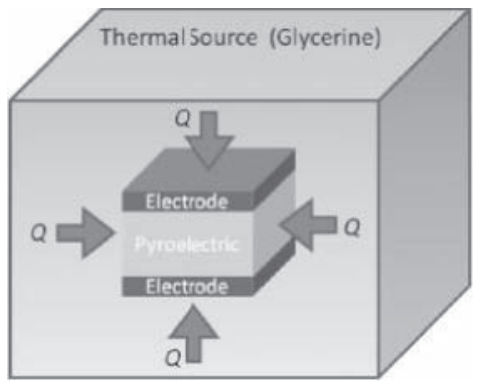

Fig. 1. Pyroelectric sensor within a heat source for measuring internal $\Delta \mathrm{S}$.

generate electrical currents proportional to temperature fluctuations. Therefore, we investigate how thermal energy is transferred from the heat source to the pyroelectric sensor. This is evaluated using heat transfer equations.

\section{A. Entropy and Heat Transfer}

In thermodynamics [2], [3], entropy, $S$, is a function of state: intrinsic and extensive. The change in $S$ is defined as the heat exchanged by the material, $\delta Q$, at a particular temperature, $T$.

$$
d S=\frac{\delta Q}{T} .
$$

To study entropy variations using a pyroelectric sensor, we consider both heat exchange and temperature variations. Thus, an entropy sensor must monitor both temperature and heat.

Heat is transferred from the environment into a pyroelectric sensor by conduction at a temperature, $T$, as illustrated in Fig. 1. This process is ruled by the general heat conduction equation for solids.

$$
\rho C_{p} \frac{\partial T}{\partial t}=\nabla \cdot(k \nabla T)+\mathrm{Q},
$$

where $\rho, C_{\mathrm{P}}$, and $k$ are the density, heat capacity at constant pressure $\left(C_{\mathrm{p}}=c_{\mathrm{p}} \cdot m\right.$, where $m$ is the mass and $c_{\mathrm{p}}$ is the specific heat capacity), and thermal conductivity of the pyroelectric material, respectively [20]; $t$ is the time, and $Q$ represents environmental heat sources. The term on the left-hand side of the equation describes time-dependent thermal variation, whereas that on the right-hand side is related to the thermal spatial gradients. From this definition, we can see that pyroelectric sensors can be used to characterize heat transfer (this observation will be clarified when studying pyroelectric response: see Eq. (3) [2], [21]).

There are certain considerations regarding the boundary conditions of this equation that should be noted:

i) Heat transfer mechanisms. In solids, heat is transferred by conduction. Convection is considered as a boundary condition at the interface if the sensor is in contact with fluids. Radiation is negligible for solid/liquid interfaces at low temperatures $(\mathrm{T}<340 \mathrm{~K})$.

ii) Space vs. time heat transfer. The contributions of both terms need to be considered. To discriminate between each term's contribution, simulations are performed.

iii) Volume vs. surface measurements. The entropy sensor can be located either inside a medium for volume entropy measurement (as illustrated in Fig. 1) or fixed to a surface

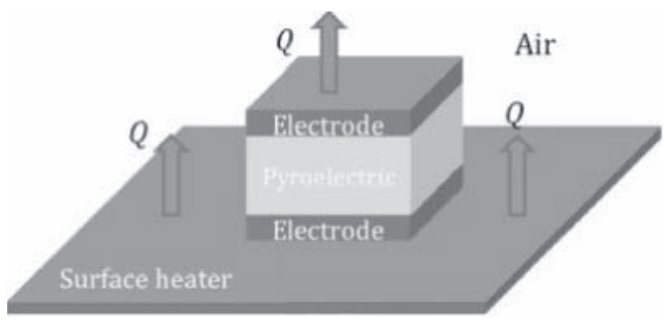

Fig. 2. Pyroelectric sensor on the surface configuration. Heat flows through the pyroelectric element along the polar axis.

for entropy flux measurement through the surface (as shown in Fig. 2). In the volume case, heat is transferred to the sensor from every direction, such that it is possible to measure entropy generation in the medium. In the case of surface sensors (to measure entropy flow through a surface), heat is conducted from one electrode plate to the other through a pyroelectric capacitor. In this case, the entropy flow is generated in the medium and transferred to the environment. In both cases, the sensor's temperature rises with heat absorption.

\section{B. Pyroelectric Response and Entropy Determination}

A pyroelectric material is a dielectric with polar point symmetry, which shows a spontaneous electrical polarization as a function of temperature. For pyroelectric flat-plate capacitors poled along the axis perpendicular to the plates, we assume a homogeneous pyroelectric material whose temperature, $T$, is uniform at any point in time; therefore, the generated pyroelectric current, $I$, is given by

$$
I=A \lambda \frac{d T}{d t},
$$

where $A$ is the electrode surface area of the material sample and $\lambda$ is the pyroelectric coefficient [22]. Integrating over time, the generated charge, $\Theta$, is

$$
\Theta=A \lambda\left(T_{f}-T_{i}\right)
$$

$T_{\mathrm{f}}-T_{\mathrm{i}}$ is the sensor temperature difference between the initial, $T_{\mathrm{i}}$, and final, $T_{\mathrm{f}}$, temperatures. From these expressions, temperature evolution can be directly inferred as

$$
\Delta T=T_{f}-T_{i}=\int \frac{I}{A \lambda} d t=\frac{\Theta}{A \lambda} .
$$

In addition, rearranging (3) we can write

$$
I=A \lambda \frac{d T}{d t}=\frac{A \lambda}{C_{\mathrm{p}}} \frac{d Q}{d t},
$$

where we consider a pure pyroelectric material with heat capacity $C_{\mathrm{p}}=\mathrm{d} Q / \mathrm{d} T$. If we assume the hypothesis of local equilibrium, entropy is defined locally and instantaneously [23]-i.e., if the pyroelectric sensor is at a particular temperature, $T$, at a particular time, $t$, then the entropy variation will be due to the heat transferred to the sensor, $\Delta Q$, as defined by (1). If this process is repeated in time, as illustrated in Fig. 3, then the total entropy variation will consist of the integral of the time intervals' variations if they are made short enough for quasistatic equilibrium. 


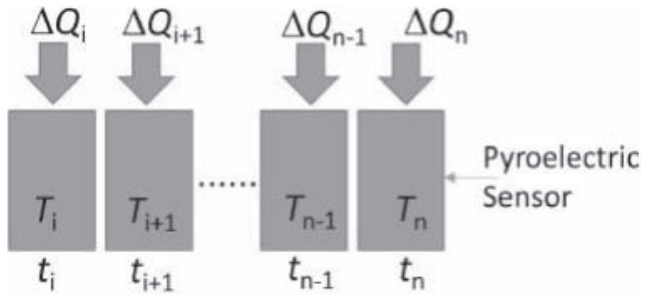

Fig. 3. Pyroelectric sensor that evolves from $T_{\mathrm{i}}$ at $t_{\mathrm{i}}$ to $T_{\mathrm{f}}$ at $t_{\mathrm{f}}$ while receiving $\Delta \mathrm{Q}$ heat over each interval, as inferred from [2].

With this approach, we can write, the entropy rate in the pyroelectric sensor for any particular interval at temperature $T$ as

$$
S_{\text {rate }}=\frac{d S}{d t}=\frac{C_{\mathrm{p}}}{A \lambda} \frac{I}{T} .
$$

If the generated current is integrated over time, entropy variation can be written as

$$
\Delta S=\int S_{\text {rate }} d t=\int \frac{C_{\mathrm{p}}}{A \lambda} \frac{I}{T} d t .
$$

Notice that $T$ is assumed to be constant over the examined intervals. While $S_{\text {rate }}$ is obtained from direct measurements of $I$ and $T, \Delta S$ can be found by numerically integrating $S_{\text {rate. }}$.

If we substitute (3) into (8), we recover the well-known thermodynamic expression

$$
\Delta S=\int \frac{\delta Q}{T}=\int_{T_{\mathrm{i}}}^{T_{\mathrm{f}}} \frac{C_{p} \mathrm{~d} T}{T}=C_{\mathrm{p}} \ln \left(\frac{T_{\mathrm{f}}}{T_{\mathrm{i}}}\right),
$$

which is used in the discussion once the experimental results allow for numerical integration.

In summary, we have considered entropy and entropy rate variations in the pyroelectric sensor as functions of its parameters. Thus, both $\Delta S$ and $S_{\text {rate }}$ can be inferred with the pyroelectric sensor.

\section{Materials And Methods}

Pyroelectric cells using two different materials with varying geometries are used. The two basic types were the commercial Polyvinylidene fluoride (PVDF) sensors depicted in Fig.4a and fabricated Lead zirconate titanate (PZT) cells. The PVDF sensors were provided by Measurement Specialties, Inc. [24]. They consist of PVDF deposited on a flexible plastic substrate. The PZT samples were fabricated using thick film technology. These cells consist of a layer of $4 \mathrm{~cm} \times 4 \mathrm{~cm}$ pyroelectric powder (Piezokeramica 856 ) sandwiched between two PdAg electrodes. Further details can be found elsewhere [25]. Cell details are described in Table 1, and the characteristics of the investigated materials are described in Table 2.

Pyroelectric sensors are usually modeled as current sources [26], such that the output current can be measured using a transresistance amplifier. The electrical circuit is depicted in Fig.5. The amplifier we use is an op-amp (TL074), and the feedback resistance, $R_{\mathrm{f}}$, is $1 \mathrm{M} \Omega$. The amplifier output voltage is measured using a computer-controlled data-acquisition system (either Agilent 34970A or

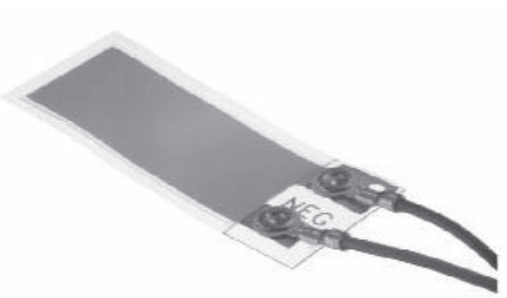

a)

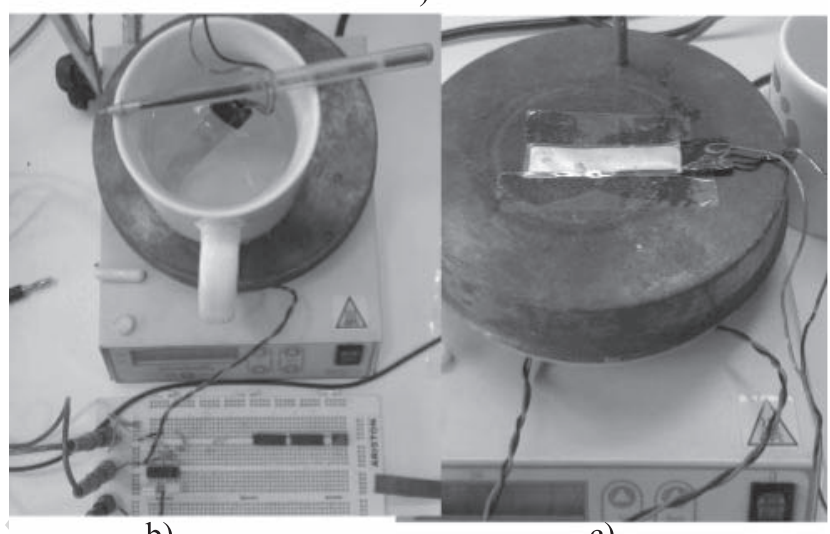

b)

c)

Fig. 4. a) Pyroelectric film from Measurement Specialties. The sensor works as a temperature and heat sensor. b) Sensor inside the glycerine (volume measurement. c) Sensor on a heating surface (surface measurement).

TABLE I

GeOMETRIC PARAMETERS OF THE INVESTIGATED PYRoELECTRIC Sensors. Further Details Can Be Found in [13]

\begin{tabular}{|c|c|c|c|c|c|}
\hline & $\begin{array}{c}\mathrm{x} \\
(\mathrm{cm})\end{array}$ & $\begin{array}{c}\mathrm{y} \\
(\mathrm{cm})\end{array}$ & $\begin{array}{c}\mathrm{z} \\
(\mu \mathrm{m})\end{array}$ & $\begin{array}{c}\text { Mass } \\
\left(10^{-5} \mathrm{~kg}\right)\end{array}$ & $\begin{array}{c}\text { Pyroelectric } \\
\text { coefficient } \lambda \\
\left(10^{-6} \mathrm{Cm}^{-2} \mathrm{~K}^{-1}\right)\end{array}$ \\
\hline PVDF1 & 17.1 & 1.2 & 28 & 10.22 & 30 \\
\hline PVDF2 & 6.20 & 1.20 & 28 & 3.71 & 30 \\
\hline PZT2 & 4.00 & 4.00 & 60 & 48.0 & 178 \\
\hline PZT4 & 4.00 & 4.00 & 100 & 80.0 & 240 \\
\hline
\end{tabular}

TABLE II

Thermal Characteristics OF THE INVestigated Materials. THIS DATA IS USED FOR THE NUMERICAL SimUlations

\begin{tabular}{|c|c|c|c|}
\hline Material & $\begin{array}{c}\text { Specific } \\
\text { heat capacity } \\
c_{\mathrm{p}}\left(\mathrm{J} \mathrm{kg}^{-1} \mathrm{~K}^{-1}\right)\end{array}$ & $\begin{array}{c}\text { Thermal } \\
\text { conductivity } \\
\left(\mathrm{W} \mathrm{m}^{-1} \mathrm{~K}^{-1}\right)\end{array}$ & $\begin{array}{c}\text { Density } \\
\left(\mathrm{kg} \mathrm{m}^{-3}\right)\end{array}$ \\
\hline $\begin{array}{c}\text { Electrode } \\
(\mathrm{Ag} / \mathrm{Pd} \mathrm{2.8:1)}\end{array}$ & 241 & 318 & 10890 \\
\hline PZT & 440 & 1.25 & 7500 \\
\hline PVDF & 962 & 0.18 & 1780 \\
\hline Alumina & 880 & 23 & 3750 \\
\hline Glycerol & 2424 & 0.289 & 1261 \\
\hline
\end{tabular}

Yokogawa DL750). The relation between the input current 201 and the output voltage is given by

$$
I=-\frac{V_{0}}{R_{\mathrm{F}}}
$$

To determine the experimental entropy variation, we conduct two different types of experiments: one measuring heat source entropy generation and the other measuring heat flow through the source's surface. In the first one, a $250-\mathrm{ml}$ 


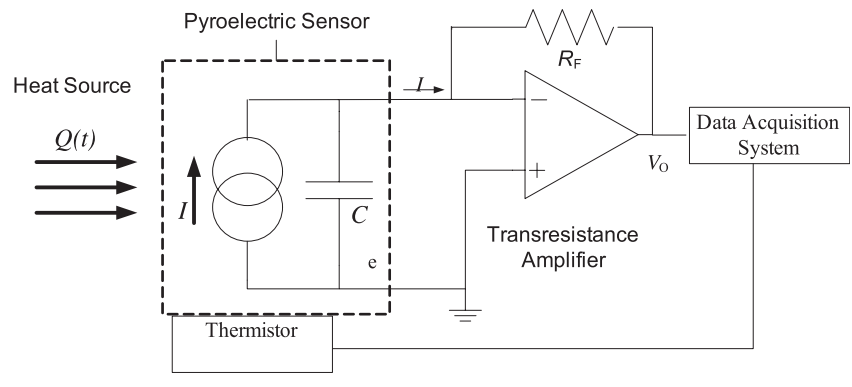

Fig. 5. Equivalent pyroelectric sensor model connected to a transresistance amplifier. The thermistor is placed so as to settle the initial temperature of the pyroelectric sensor in the environment while the pyroelectric temperature monitoring is carried out directly based on its current profile [15].

glycerin thermal bath at $343 \mathrm{~K}\left(70{ }^{\circ} \mathrm{C}\right)$ is prepared using a heater Ovan - MicroMagmix as the heat source. The samples, originally at room temperature, are dipped into the bath. Glycerin is used because it is a good electrical insulator and its boiling point is $563 \mathrm{~K}$ (Fig. 4b). In the second experiment, we attach the pyroelectric sensor to a metallic surface that acts as a heat source whose temperature is set at $343 \mathrm{~K}$, as illustrated by the surface heater in Fig. 4c.

The physical structures of the pyroelectric film (see Fig. 4, schematically illustrated in Figs 1-2) with the data described in Table 1 are modeled using finite-element software (Comsol Multiphysics). One-dimensional (1D) thermal simulations are performed using the data in Table 1, and, taking advantage of sample symmetries along the z-axis, they can be extrapolated to 3D. Border effects are neglected, which is a common approximation for planar capacitors. Moreover, metal electrodes are better thermal conductors than dielectric materials; therefore, they do not limit heat transfer from the heat source to the pyroelectric material. Thermal simulations consider a heat source at constant temperature (a glycerin bath at the experimental temperature), with the pyroelectric sensor being dipped at an initial temperature equal to the ambient temperature. Thus, temperature, enthalpy, and entropy evolutions are monitored in the capacitor structure with consideration of the metallic electrodes. The electrical response of the pyroelectric sensor is not simulated. Similarly, the experimental setup for the surface measurement illustrated in Fig. $4 \mathrm{c}$ is modeled considering a surface temperature source and air as the environment. Convection is not considered.

\section{Results}

In this section, we present the experimental results for the electrical responses of the different pyroelectric sensors when exposed to various heating conditions. These results are compared with simulation analyses.

\section{A. Heat Measurements in Dipped Samples}

Heat is related to pyroelectric current by (3) using the $C_{\mathrm{p}}$-value for the sensor. Enthalpy, $H$, is the parameter investigated in simulations $(H=U+P \cdot V$, where $U$ is the internal energy, $P$ is the pressure, and $V$ is the volume). If no external work is done on the solids, the enthalpy equals heat variation, which is the case for these experiments. The comparison

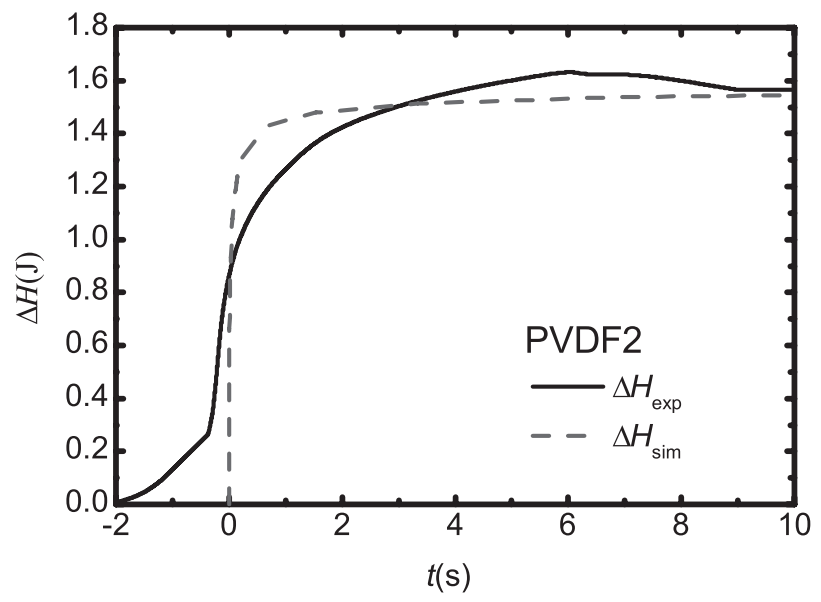

Fig. 6. Enthalpy variation in the PVDF2 sensor for a temperature change of $45 \mathrm{~K}$. Comparison between experimental results and simulation shows good agreement.

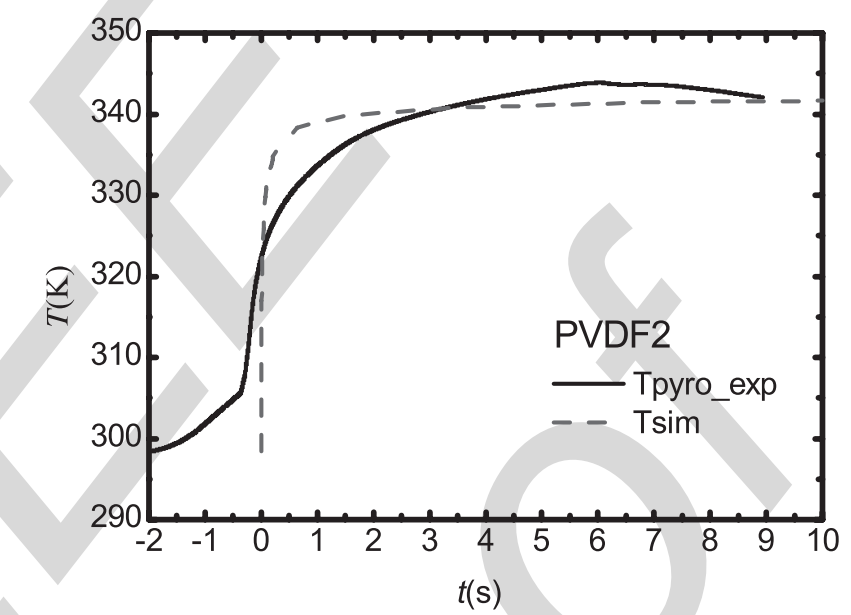

Fig. 7. Experimental and simulated temperature profiles in the PVDF2 sensor obtained using (5).

between the experimental results and the simulation curve is presented in Fig. 6. There is good agreement, though slight differences are found in the transient dynamic process; these differences arise from the practical setup of dipping the sample in the glycerin bath.

\section{B. Temperature Measurement in Dipped Samples}

The temperature in the pyroelectric sensor is inferred from (5). Experimental results for the PVDF2 sample compared with the pyroelectric temperature obtained from the simulation based on the heat transfer equation are presented in Fig. 7. The pyroelectric sensor follows the expected temperature variation as described in thermometry references [16], [18]. This avoids the need for external temperature sensors with different time constants, which cannot measure the pyroelectric's inner temperature.

\section{Entropy Determination in Dipped Samples}

Entropy is immediately obtained from heat and temperature using (9). The experimental results are compared with the simulation curves, and there is good agreement (see Fig. 8). 


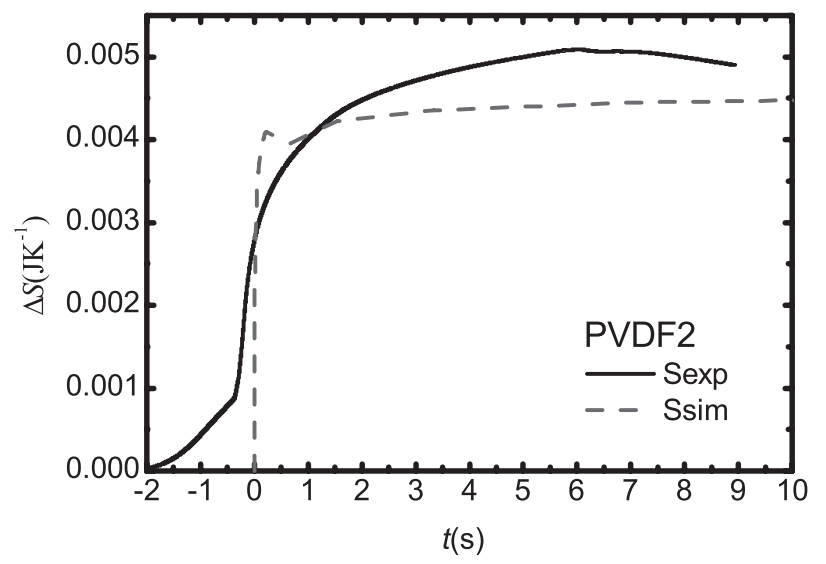

Fig. 8. Experimental and simulated entropy-generation in the PVDF2 sensor.

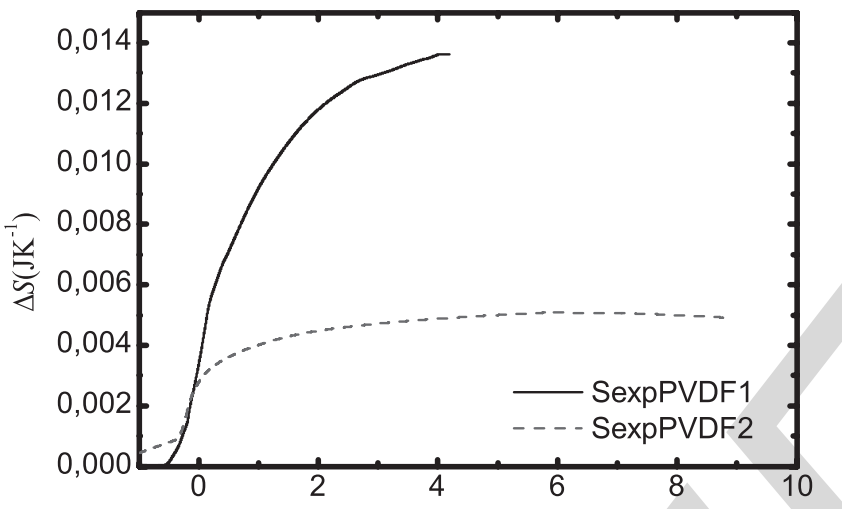

$t(\mathrm{~s})$

Fig. 9. Measured entropy variation for PVDF sensors with two different volumes.

\section{Geometry Comparison in Dipped Samples}

Entropy is an extensive property; thus, it scales with volume. We compare the entropy generation of two PVDF sensors of various sizes (PVDF1 and PVDF2 in Table 1). Their volume ratio is 2.76 . Their experimental entropy ratio after heating is approximately 2.7 , which perfectly matches the volume ratio, as illustrated in Fig. 9.

\section{E. Surface Measurements}

The relationship between the entropy flux and volume measurements is investigated according to the setups described in Figs. 1 and 2. The expected differences are found at the maximum entropy change (see Fig. 10). Experimental results illustrated in Fig.11 show good agreement with the simulation for the total $\Delta S$ but larger differences during the dynamic response of the sensor. These deviations are discussed later.

\section{Discussions}

\section{A. Model and Approximations}

In this section, we first discuss the impact of the assumptions used in heat transfer equation (2); later, we evaluate the experimental results according to thermodynamics and simulation.

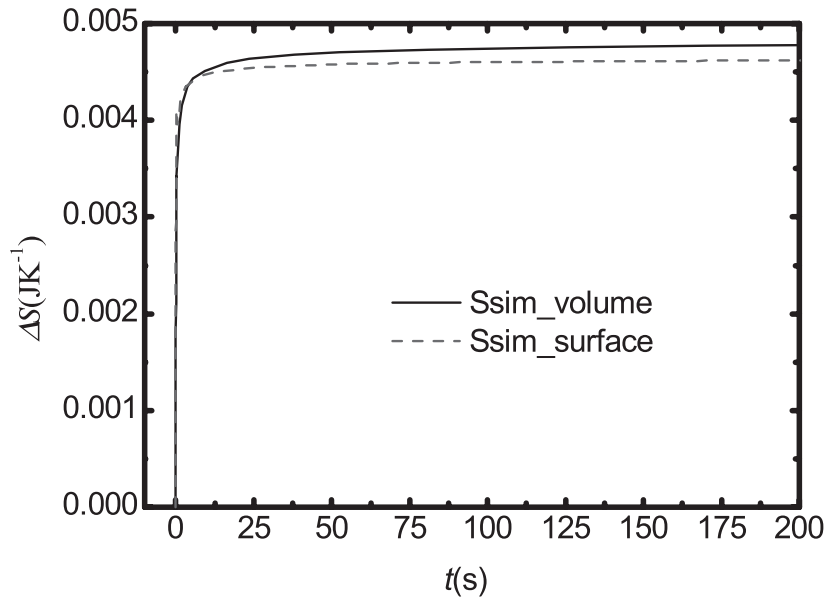

Fig. 10. Simulations of entropy generation in the pyroelectric sensor PVDF2 for two cases: 1) surface measurement, where one side of the sensor is at room temperature because of contact with air (red dashed line) and 2) volume measurement, where the sensor is completely inside the glycerin bath (black solid line).

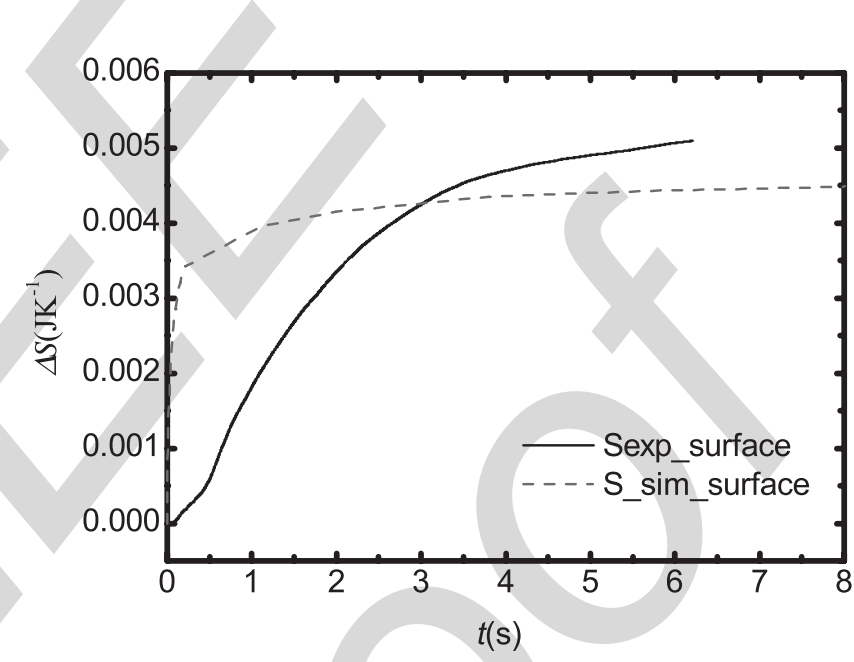

Fig. 11. Comparison between experimental and simulated entropy flux for the surface measurements.

Heat convection may have had an effect on the pyroelectric measurements because the samples were dipped into the bath. To evaluate this effect, we evaluate Biot's number, defined as $\mathrm{Bi}=h \cdot L / k$, where $h$ is the convective parameter (estimated as approximately 15 ), $k$ is the thermal conduction, and $L$ is the thickness of the sample [20]. In our case, $\mathrm{Bi}=0.05$. As long as $\mathrm{Bi}<0.1$, thermal conduction is fast enough to minimize thermal gradients; thus, convection is negligible.

In heat transfer equation (2), heat is transferred conductively in accordance with $k \cdot \nabla^{2} T$. The temperature gradient is found to be linear inside the pyroelectric sensor, both for the volume and surface simulations. Thus, the second-order temperature derivative is zero, and this term does not contribute. Consequently, time-dependent heat transfer occurs, which is convenient for pyroelectric conversion and entropy estimation, as inferred from the comparison of (2) and (3). However, pyroelectric equation (3) assumes that temperature is homogenous inside the pyroelectric, whereas 


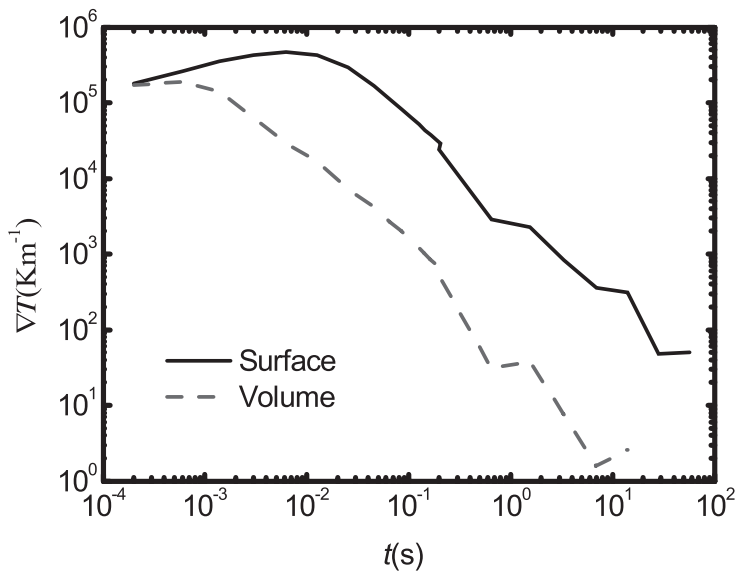

Fig. 12. Simulation of the thermal gradient magnitude-evolution inside the sensor for volume and surface measurements.

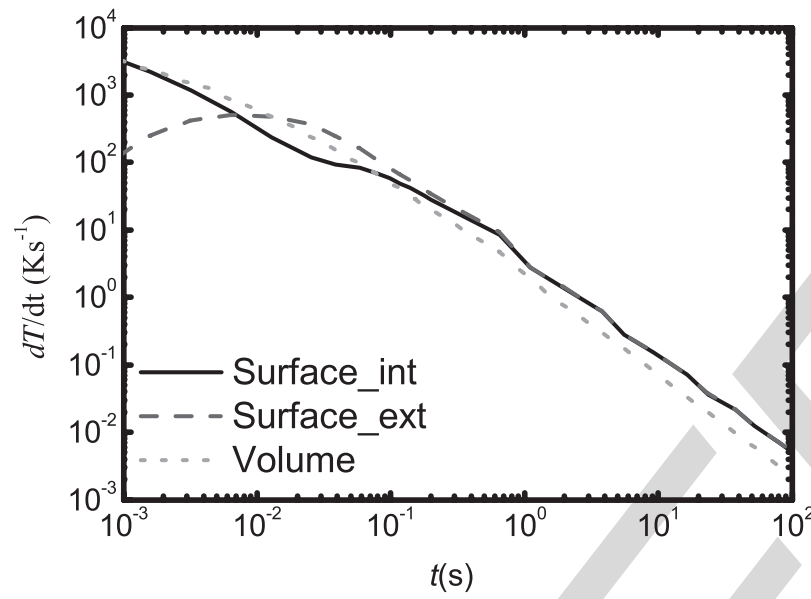

Fig. 13. Simulated temperature derivative, dT/dt, for the surface measurement at the hot side (surface_int), at the cold side (surface_ext), and for the volume measurement (volume). the gradient is time-dependent, as illustrated in Fig. 12. In the model described in Section II, the sensor is approximated as a lumped system; that is, during transient heat transfer, the temperature does not vary with position because of the small dimensions and high conductivity terms and small gradients, but the system temperature varies uniformly with time. This approximation is found to be valid for times greater than $0.1 \mathrm{~s}$ after the initial dipping; however, in the initial transient heat transfer, temperatures must be homogenized, as obtained from simulations and illustrated in Fig.13, where the temperatures at both surfaces of the pyroelectric and the average temperature converge. Larger differences should be observed for surface measurements, which explain the transient differences between simulations and experimental results in Fig.11. For this case, improved analyses of the pyroelectric coefficient, $\lambda$, as a function of position may improve agreement. Finally, we assume that both thermal capacity, $C_{\mathrm{p}}$, and the pyroelectric coefficients, $\lambda$, are temperature-independent in this first study. This is a reasonable approximation, considering the good agreement between the thermodynamic model and the experimental results. Future studies attempting to characterize $C_{\mathrm{p}}(T)$ will be necessary to take this second-order effect into account.

\section{B. Results and Thermodynamics Model}

Results comparing sensor geometries show that we can characterize entropy generation, which is an extensive parameter, if volume is normalized and thus becomes independent of the sensor geometry. Thus, whatever the sensor geometry is, realistic values can be achieved in environmental measurements, though the sensor should be as small as possible in order to not affect the environmental temperature. In our experiments, the environment is the thermal bath heat source. Good agreement between volume and surface measurements was found, although the transient evolution model should be improved for better agreement in the transient regions (Fig. 11). Surface measurements may be improved using a specific sensor for entropy measurements, thereby minimizing the transient discrepancy.

Entropy-generation in the PVDF sample increases when dipped in the thermal source, in accordance with (9), as illustrated in Fig. 8 and compared in Table 2. The real interest of this work, however, is to relate the sensors' entropy to environmental entropy generation; thus, it is convenient to validate these results using thermodynamic models. We can estimate the theoretical thermodynamic entropy for the thermal bath from (11) and (12). While the samples reach the bath temperature, the bath, which is considered to bea heat source, undergoes a change $\Delta T<0.2 \mathrm{~K}$.

$$
\Delta S=\frac{\Delta Q}{T}=\frac{C_{p} \Delta T}{T}=\frac{C_{\mathrm{p}} \cdot\left(T_{\mathrm{f}}-T_{\mathrm{i}}\right)}{T_{\mathrm{i}}},
$$

where

$$
T_{\mathrm{f}}=\frac{\sum_{i} C_{\mathrm{p}, \mathrm{i}} \cdot T_{\mathrm{i}}}{\sum_{\mathrm{i}} C_{\mathrm{p}, \mathrm{i}}},
$$

and the sum is calculated for all of the elements involved in the problem (heat source and sensors in this case). The pyroelectric sensor's temperature is not constant; thus, the entropy variation is given by

$$
\Delta S=\int \frac{\delta Q}{T}=\int_{T_{\mathrm{i}}}^{T_{\mathrm{f}}} \frac{C_{p} \mathrm{~d} T}{T}=C_{\mathrm{p}} \ln \left(\frac{T_{\mathrm{f}}}{T_{\mathrm{i}}}\right),
$$

which is compared with the experimental data obtained through numerical integration of the experimental results using

$$
\begin{aligned}
\Delta S & =\int \frac{C_{\mathrm{p}}}{A \lambda} \frac{I}{T} d t=\int \frac{C_{\mathrm{p}}}{A \lambda} \frac{I}{\left(T_{i}+\int \frac{I}{A \lambda} d t\right)} d t \\
& \approx \frac{C_{\mathrm{p}}}{A \lambda T_{\mathrm{i}}} \int I\left(1-\frac{\int \frac{I}{A \lambda} d t}{T_{\mathrm{i}}}\right) d t \approx \frac{C_{\mathrm{p}} \Theta}{A \lambda T_{i}}\left(1-\frac{\frac{\Theta}{A \lambda}}{T_{i}}\right),
\end{aligned}
$$

as inferred from (5) and (9).

The comparison of the predictions of this thermodynamic model with the experimental results achieved with equations $7-14$ for the investigated samples is shown in Table 3. To compare different materials, we take the data for 
TABLE III

COMPARISON BETWEEN DyNAMiC SimUlations, THERMOdynamic Models and EXPERIMENTAL Results IN Volume (GLYCERINe) AND SuRface (AIR) MEASUREMENTS

\begin{tabular}{|l|l|l|l|l|l|l|}
\hline & & \multicolumn{1}{|c|}{$\begin{array}{c}\text { Thermodynami } \\
\text { cs model }\end{array}$} & \multicolumn{2}{|c|}{ Experimental } \\
\hline & $\begin{array}{l}\text { Heat } \\
\text { Sourc } \\
\mathrm{e}\end{array}$ & $\begin{array}{l}\Delta \mathrm{T} \\
(\mathrm{K})\end{array}$ & $\begin{array}{l}\mathrm{H} \\
(\mathrm{J})\end{array}$ & $\begin{array}{l}\Delta \mathrm{S} \\
\left(\mathrm{JK}^{-1}\right)\end{array}$ & $\mathrm{H}(\mathrm{J})$ & $\begin{array}{l}\Delta \mathrm{S}\left(\mathrm{JK}^{-1}\right. \\
)\end{array}$ \\
\hline PVDF1 & $\begin{array}{l}\text { Glyce } \\
\text { rin }\end{array}$ & 45 & 4.42 & 0.0138 & 4.36 & 0.0133 \\
\hline PVDF2 & $\begin{array}{l}\text { Glyce } \\
\text { rin }\end{array}$ & 45 & 1.60 & 0.00501 & 1.58 & 0.00504 \\
\hline PVDF1[25] & Air & 68 & 2.85 & 0.0202 & 2.90 & 0.0203 \\
\hline PVDF2[25] & Air & 80 & 6.69 & 0.00848 & 6.94 & 0.00832 \\
\hline PZT1[25] & Air & 30 & 15.23 & 0.049 & 12.91 & 0.040 \\
\hline PZT4[25] & Air & 28 & 15.23 & 0.049 & 15.4 & 0.049 \\
\hline
\end{tabular}

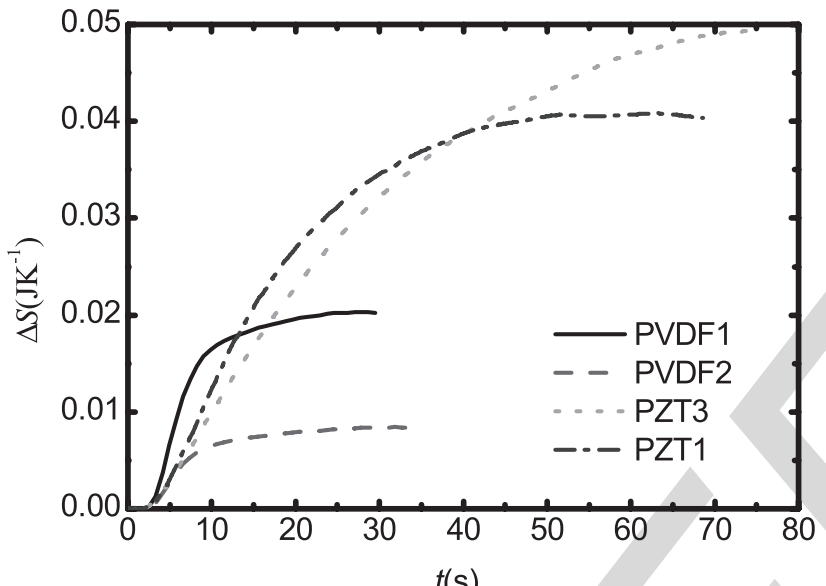

$t(\mathrm{~s})$

Fig. 14. Comparison between entropy generation in PVDF and PZT heated with air flow in accordance with data from [25]. As illustrated in Table 3, experimental results agree with the thermodynamic model, regardless of the material used (PVDF vs. PZT) or the heating source (air flow vs. glycerin bath).

PZT from [25]. In that case, samples were heated with air flow; the results are illustrated in Fig. 14. The comparison between the obtained enthalpies and entropies for different temperature excursions also shows good agreement with experimental results, as depicted in Table 3. These results are also independent of the type of pyroelectric sensor (PVDF vs. PZT) and even the heat source (glycerin bath vs. air flow), demonstrating the effective behavior of the entropy sensor.

\section{Beyond the Sensor Entropy Measurement: The Environmental Measurement}

Up to this point, we have discussed the entropy accumulated in the pyroelectric sensor. The interesting parameter, however, is the entropy released by the heat source, which is given by (11) and (12) for the case in which the $C_{\mathrm{p}}$ of the heat source is known. In our experimental setup for the glycerin bath, $\Delta S_{\mathrm{hs}}=-4.68 \cdot 10^{-3} \mathrm{~J} / \mathrm{K}$. The difference between the entropy released by the heat source, $\Delta S_{\mathrm{hs}}$, and that absorbed by the sensor, $\Delta S_{\text {sensor }}$ (PVDF2 sample), is the entropy gained by the Universe, $\Delta S_{\text {uni }}=\Delta S_{\mathrm{hs}}-\Delta S_{\text {sensor. }}$. According to the second principle of thermodynamics, $\Delta S_{\text {uni }}>0$ for irreversible processes, and $\Delta S_{\text {uni }}=0$ for reversible processes. We find that $\Delta S_{\text {uni }}=8.62 \cdot 10^{-3} \mathrm{~J} / \mathrm{K}$ and $0.37 \cdot 10^{-3} \mathrm{~J} / \mathrm{K}$ for PVDF1 and PVDF2, respectively. Though $\Delta \mathrm{S}_{\text {uni }}>0$ in both cases, indicating that they are irreversible, the overall measurement accuracy must be improved for exact heat source entropy determination. This result is of particular interest for various reasons. First, because the size of the sensor is related to entropy generation, it should be kept small (note that $\Delta S_{\text {uni }}$ is larger for PVDF1 than for PVDF2). Second, in the glycerin bath, a simple thermodynamic model is easily obtained and comparison between $\Delta S$ for the sensor and heat source is straightforward; however, in the case of air flow, the model is much more complex. However, the entropy generation at the sensor is a good estimation for both heating sources, i.e., the thermal bath and the surface heater. In fact, for PVDF2, the sensor entropy is $5.04 \cdot 10^{-3} \mathrm{~J} / \mathrm{K}$ and the generated entropy is $\Delta S_{\text {uni }}=0.37 \cdot 10^{-3} \mathrm{~J} / \mathrm{K}$. The difference between the sensor and the heat source entropy is $\sim 7.3 \%$, which could be reduced even more by scaling down the sensor size, with a limit imposed by the second principle. Also, we have kept a surface at room temperature. This sensor could be placed in an isolated environment to improve measurements.

\section{CONCLUSION}

We have investigated pyroelectric materials as entropy sensors and proved the concept from these results. Heat and temperature are estimated, and, thus, entropy is inferred. Geometry-dependence did not affect normalized entropy determination, as was theoretically expected.

Both volume entropy and entropy flow were measured. Whereas volume measurements showed good agreement with theoretical estimations, surface measurements only provided a good estimation of the total entropy flow, and transient entropy evolution should be investigated in more detail. Finally, the changes in the heat source and universe entropy could be obtained with our sensor using convenient thermodynamic models, which should provide insights into system energy yield.

Our accuracy was around $10 \%$, which is encouraging for this initial stage of testing, because the sensor was able to predict the general trend even though the system has not yet been optimized. Possible avenues to improve these sensors include designing a pyroelectric sensor that minimizes geometrical dimensions and substrate effects with improved thermal conduction while maintaining a small thermal capacity.

These sensors have possible applications in characterization of both electrochemical processes and irreversible thermodynamic processes, like combustion, heat engines, and electrical systems with Joule dissipation.

\section{REFERENCES}

[1] A. Navid, D. Vanderpool, A. Bah, and L. Pilon, "Towards optimization of a pyroelectric energy converter for harvesting waste heat," Int. J. Heat Mass Transf., vol. 53, nos. 19-20, pp. 4060-4070, Sep. 2010.

[2] A. Bejan, Advanced Engineering Thermodynamics, 2nd ed. Hoboken, NJ, USA: Wiley, 2006.

[3] M. W. Zemansky and R. H. Dittman, Heat and Thermodynamics. New York, NY, USA: McGraw-Hill, 1990. 
[4] C.-J. Ding and L.-F. Luo, "Experimental study of entropy production in cells under alternating electric field," Chin. Phys. Lett., vol. 29, no. 8, p. 088701, Aug. 2012.

[5] V. Basso, F. Russo, J.-F. Gerard, and S. Pruvost, "Direct measurement of the electrocaloric effect in poly(vinylidene fluoride-trifluoroethylenechlorotrifluoroethylene) terpolymer films," Appl. Phys. Lett., vol. 103, no. 20, p. 202904, 2013.

[6] X. Yuan and F. Yang, "Energy transfer in pyroelectric material," in Heat Conduction-Basic Research, V. S. Vikhrenko, Ed. Beijing, China: InTech, 2011, pp. 229-248.

[7] Z. Jiang, J. Zhang, L. Dong, and J. Zhuang, "Determination of the entropy change of the electrode reaction by an ac electrochemicalthermal method," J. Electroanal. Chem., vol. 469, no. 1, pp. 1-10, Jun. 1999.

[8] M. Mansour, N. Chokani, A. I. Kalfas, and R. S. Abhari, "Time-resolved entropy measurements using a fast response entropy probe," Meas. Sci. Technol., vol. 19, no. 11, p. 115401, Nov. 2008.

[9] A. Eddahech, O. Briat, and J.-M. Vinassa, "Lithium-ion battery heat generation investigation based on calorimetric entropy measurements," in Proc. IEEE Int. Symp. Ind. Electron., May 2013, pp. 1-5.

[10] Y. Troxler et al., "The effect of thermal gradients on the performance of lithium-ion batteries," J. Power Sour., vol. 247, pp. 1018-1025, Feb. 2014.

[11] R. A. Huggins, Energy Storage. New York, NY, USA: Springer, 2010.

[12] M. Naderi and M. M. Khonsari, "Thermodynamic analysis of fatigue failure in a composite laminate," Mech. Mater., vol. 46, pp. 113-122, Mar. 2012.

[13] A. Cuadras, V. J. Ovejas, and M. Quilez, "Entropy as a wear out indicator: The resistor example," in Proc. 10th Int. MultiConf. Syst., Signals Devices (SSD), Hammamet, Tunisia, 2013, pp. 1-5.

[14] V. J. Ovejas and A. Cuadras, "Battery state of charge determination from heat and entropy measurements," in Proc. 12th Int. Conf. Energy Storage, Lleida, Spain, 2012.

15] A. Cuadras and V. J. Ovejas, "Pyroelectric sensor for entropy measurements," in Proc. IEEE 11th Int. Multi-Conf. Syst., Signals Devices (SSD), Feb. 2014, pp. 1-5.

[16] C. F. Tsai and M. S. Young, "Pyroelectric infrared sensor-based thermometer for monitoring indoor objects," Rev. Sci. Instrum., vol. 74, no. 12 , p. $5267,2003$.

[17] A. Rogalski, "Infrared detectors: Status and trends," Prog. Quantum Electron., vol. 27, nos. 2-3, pp. 59-210, 2003.

[18] P. R. N. Childs, J. R. Greenwood, and C. A. Long, "Review of temperature measurement," Rev. Sci. Instrum., vol. 71, no. 8, p. 2959, 2000.

[19] S. B. Lang and S. Muensit, "Review of some lesser-known applications of piezoelectric and pyroelectric polymers," Appl. Phys. A, Mater. Sci. Process., vol. 85, no. 2, pp. 125-134, Nov. 2006.

[20] A. F. Mills, Heat Transfer, 2nd ed. Upper Saddle River, NJ, USA: Prentice-Hall, 1998.
[21] S. B. Lang, "Pyroelectricity: From ancient curiosity to modern imaging tool," Phys. Today, vol. 58, no. 8, pp. 31-36, 2005.

[22] Y. Xu, Ferroelectric Materials and Their Applications. Amsterdam, The Netherlands: Elsevier, 1991.

[23] G. Lebon, D. Jou, and J. Casas-Vázquez, Understanding Nonequilibrium Thermodynamics: Foundations, Applications, Frontiers. New York, NY, USA: Springer, 2008.

[24] (Jul. 2014). Measurement Specialties. [Online]. Available: http://www.meas-spec.com/

[25] A. Cuadras, M. Gasulla, and V. Ferrari, "Thermal energy harvesting through pyroelectricity," Sens. Actuators A, Phys., vol. 158, no. 1, pp. 132-139, 2010.

[26] V. Ferrari, D. Marioli, and A. Taroni, "Displacement sensor based on pyroelectric thick films and contactless light-spot cursor," IEEE Trans. Instrum. Meas., vol. 51, no. 4, pp. 819-823, Aug. 2002.

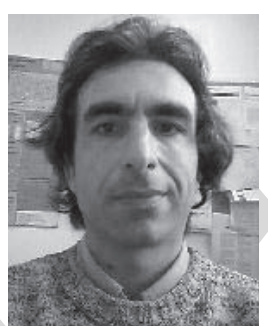

Angel Cuadras was born in Tarragona in 1974. $\mathrm{He}$ received the B.S., M.S., and Ph.D. degree in physics from the University of Barcelona, in 1997, 1999, and 2002, respectively. He was involved in the technology and growth of $\mathrm{Si}_{1-x-y} \mathrm{Ge}_{x} \mathrm{C}_{y}$ materials and the physical and electrical characterization of MOS capacitors. In 2003, he joined the Instrumentation, Sensors, and Interfaces Group, Universitat Politècnica de Catalunya, where he is currently an Associate Professor. His main interests are energy systems monitoring (with a major focus on battery state of health and state of charge) and characterization of systems using impedance spectroscopy and entropy characterization. He is a member of the IEEE Instrumentation and Measurement Society. He was the Chair of the IEEE 11th Multi-Conference on Signals, Systems, and Devices (2014).

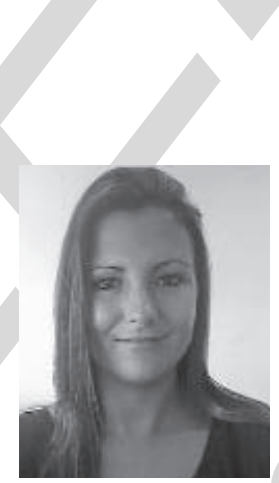

Victoria Julia Ovejas was born in Barcelona in 1985. She received the B.S. and M.S. degrees in telecommunications engineering from the Universitat Politècnica de Catalunya (UPC), in 2008 and 2010, respectively, where she is currently pursuing the Ph.D. degree in electronics engineering. She is a member of the Instrumentation, Sensors, and Interfaces Group with UPC. Her main interests are Li-ion battery monitoring (including state of charge and state of health) and energy harvesting-related subjects. 


\section{AUTHOR QUERIES}

$\mathrm{AQ}: 1=$ Please check whether the edits made in the financial section are OK.

AQ:2 = Please provide the year of the IEEE sensors conference.

AQ:3 = Please provide the page range for ref. [14].

AQ:4 = Please note that references [18] and [27] are the same, hence we deleted Ref. [27]. This change will also reflect in the citations present in the body text. Please confirm. 